\title{
Dielectric-barrier-discharge vortex generators: characterisation and optimisation for flow separation control
}

\author{
Timothy N. Jukes $\cdot$ Kwing-So Choi
}

Received: 29 May 2011/Revised: 17 August 2011/Accepted: 24 September 2011/Published online: 17 November 2011

(C) Springer-Verlag 2011

\begin{abstract}
We investigated the use of dielectric-barrierdischarge plasma actuators as vortex generators for flow separation control applications. Plasma actuators were placed at a yaw angle to the oncoming flow, so that they produced a spanwise wall jet. Through interaction with the oncoming boundary layer, this created a streamwise longitudinal vortex. In this experimental investigation, the effect of yaw angle, actuator length and plasma-induced velocity ratio was studied. Particular attention was given to the vortex formation mechanism and its development downstream. The DBD plasma actuators were then applied in the form of co-rotating and counter-rotating vortex arrays to control flow separation over a trailing-edge ramp. It was found that the vortex generators were successful in reducing the separation region, even at plasma-to-freestream velocity ratios of less than $10 \%$.
\end{abstract}

$\begin{array}{ll}\text { Abbreviations } \\ \text { CoR } & \text { Co-rotating } \\ \text { CtR } & \text { Counter-rotating } \\ \text { DBD } & \text { Dielectric-barrier-discharge } \\ \text { PIV } & \text { Particle image velocimetry } \\ \text { VG } & \text { Vortex generator }\end{array}$

Electronic supplementary material The online version of this article (doi:10.1007/s00348-011-1213-0) contains supplementary material, which is available to authorized users.

T. N. Jukes $(\bowtie) \cdot$ K.-S. Choi

Faculty of Engineering, University of Nottingham,

University Park, Nottingham NG7 2RD, UK

e-mail: Timothy.Jukes@nottingham.ac.uk

K.-S. Choi

e-mail: Kwing-So.Choi@nottingham.ac.uk

\section{Introduction}

Flow separation control by longitudinal vortices has been a subject of study for many years. The concept and first application seems to have been by Taylor (1947) using relatively simple vane-type vortex generators, devices that have found many practical applications on commercial aircraft and in industry. Vortex generators (VGs) prevent flow separation by creating an array of streamwise vortices close to the surface of an aerodynamic body. This increases the mixing between the boundary layer and the free steam, so that high momentum fluid is brought from the outer flow into the near wall region. This re-energises the near wall fluid, allowing it to withstand more severe adverse pressure gradients. Flow separation can then be delayed, controlled or completely avoided. See Pearcey (1961), Bushnell (1992) and Lin (2002) for reviews of different types of vortex generators and flow control applications.

Vane-type VGs are the most widely used devices and consist of a row of small plates that protrude normal to the body at a small incidence to the flow, so that the pressure difference between the plate surfaces leads to a longitudinal vortex from the tip. There are a huge number of different designs of vane-type VGs (Schubauer and Spangenberg 1960; Lin 2002), which can be broadly categorised by whether they create co-rotating (CoR) or counter-rotating $(\mathrm{CtR})$ vortex pairs. For CoR-VGs, flow control is effective only when the vortices have large enough spanwise spacing to prevent unfavourable interactions between adjacent vortices (i.e. the upwash from one vortex is not disturbed by the downwash from an adjacent vortex). This occurs when the initial vortex spacing is greater than about three times their height (Pearcey 1961). In addition, CoR-VGs tend to generate vortices that remain close to the wall as they travel downstream, but displace 
laterally under the velocity induced by the vortex images in the wall. In contrast, CtR-VGs typically have stronger initial effects since the vortices combine to give larger induced upwash and downwash motions. However, the vortices do not persist for as long downstream since the vortex pairs have more substantial interactions than for CoR systems. The effectiveness of CtR-VGs largely depends on whether the vortices are arranged to give common-flow up or common-flow down between the vortex pairs. Pearcey (1961) observed that for common-flow up configurations, the vortices initially move closer together and then lift away from the wall. This can cause the vortex pairs to lift outside of the boundary layer region, so that they are no longer effective at re-energising the boundary layer fluid, although the amount of lifting depends somewhat on the external pressure gradient (Godard and Stanislas 2006a). In common-flow down configurations, the vortices initially move laterally apart whilst remaining close to the surface until the vortices from one pair begin to interact with the vortices from adjacent pairs. This creates new common-flow up arrangements that then lift from the surface and hinder the momentum transfer process. Detailed measurements of the flow structure of streamwise vortices and vortex pairs have been taken by Shabaka et al. (1985), Mehta and Bradshaw (1988), Pauley and Eaton (1988), and Angele and Grewe (2007) and analysed numerically by You et al. (2006).

The success of vane-type VGs largely stems from the fact that streamwise vortices are characterised by remarkable organisation and longevity (Bushnell 1992), persisting for over $100 h$ downstream (Pearcey 1961). Plus they are cheap, simple and can be retrofitted to problem areas. Successful designs typically have vane height of the order of the boundary layer thickness, $h=\delta$, although recent work concentrates on much smaller devices with $h \sim 0.1-0.5 \delta$ to try to minimise the device drag (so-called micro-VGs, see Lin 2002). Recently Godard and Stanislas (2006a) optimised the VG geometry over a specially designed bump to mimic an airfoil on the verge of separation. They found triangular vanes with height $h \sim 0.37 \delta$, length $l=2 h$, angle of incidence $\beta=18^{\circ}$ and spanwise spacing $\lambda=6 h$ performed best. They also found that $\mathrm{CtR}$ configurations were more efficient than $\mathrm{CoR}$ by a factor of two.

However, the main problem with vane-type VGs is that they have an inherent drag penalty. Unfortunately, this drag penalty still exists when the flow control is no longer required (unless they are mechanically retracted, which adds design complexity). For example, in applications on aircraft wings, the VGs are only usually necessary during take-off and landing, so the VGs can cause significant drag penalty over the cruise part of the flight envelope. It is therefore of interest to investigate the means of generating streamwise vortices that can be switched on and off without drag penalty when not required. One way to achieve this goal is by vortex generator jets. Small holes or slots are machined into the surface from which a jet is blown. To be efficient, such jets must be pitched to the surface and skewed to the main flow direction, so that a longitudinal vortex is generated in the boundary layer by the interaction between the jet and the cross-flow (Godard et al. 2006; Godard and Stanislas 2006b). The principle of flow control is the same as for vane-type VGs: momentum transfer between the free-stream and the near-wall region. Johnston and Nishi (1990) appear to be the first to demonstrate that VG jets do indeed generate streamwise vortices and also showed that they could reduce turbulent flow separation when the jet-to-free-stream velocity ratio was above 0.8 . Compton and Johnston (1992) showed that the vortices produced were similar to vane-type VGs, but decayed faster downstream. Khan and Johnston (2000) made crossstream velocity measurements and showed that the vortices were produced by the sweep of the cross-flow around the upper side of the jet. In addition, Zhang (1999, 2000, 2003) studied the development of CtR- and CoR-VG jets and the near-field of CoR-VG jets. A complicated flow structure was found with horseshow vortices in front of the nozzles and counter-rotating vortices immediately behind, which developed into a single streamwise vortex downstream. A review of these and other works can be found in Johnston (1999).

The DBD plasma actuator that we used in this study creates a localised region of weakly ionised plasma close to the aerodynamic surface. This produces a body force generating a wall jet that can be used to control the flow (Moreau 2007). These DBD plasma actuators are purely electrical devices, so they can be rapidly turned on and off as required. They can be flush-mounted or manufactured into the surface, so there should be no drag penalty when they are not being used. Unlike VG jets, DBD plasma actuators do not need ducting or holes. The DBD plasma actuators have, therefore, huge potential for aerospace applications and have received much attention recently (Corke et al. 2010).

These actuators have been successfully used in boundary layers (Corke et al. 2010; Moreau 2007; Grundmann and Tropea 2008), as well as around bluff bodies (Jukes and Choi 2009a, b, c, d). However, the majority of studies have used actuators that produce a body force in the streamwise direction (i.e. co-flow forcing using a DBD along the span). Whilst this can be very effective if used close to the separation point, where long-lasting global modification to the wake structure can be produced with very little energy input (Jukes and Choi 2009c), flow separation is usually three dimensional and changes the location with time. We are therefore interested in DBD 
actuators that cover some streamwise distance as these might be more versatile in unsteady, 3D or turbulent flows. Here, we use streamwise-oriented and yawed actuators to induce a spanwise component of body force. This concept can be traced back to 1998 (Roth et al. 1998), although the configuration was counterproductive in their study, so that the subject received little further attention. However, more recently, Jukes et al. (2006) and Choi et al. (2010) used two rows of streamwise DBD actuators in a turbulent boundary layer which alternately produced a spanwise force. Their aim was to create a spanwise oscillation of near-wall fluid to reduce skin-friction drag, similar to a mechanically oscillating wall (Choi et al. 1998). Whilst the skin friction did appear to be reduced, the DBD actuators created streamwise vortices in addition to the oscillating flow. This inspired the authors to use spanwise DBD forcing to generate streamwise vortices for flow separation control (Okita et al. 2008), where they demonstrated that a single, yawed DBD actuator could generate a large-scale streamwise vortex which could significantly delay separation over a NACA 0024 airfoil. Also of interest here are recent thrust vectoring studies by Porter et al. (2009), Bolitho and Jacob (2008) and Benard et al. (2008) who used two opposing plasma actuators, arranged so that the tangential jets from each collide to produce a wall-normal jet (see also Segawa et al. 2009). These authors demonstrated that by varying the induced force on one of the electrodes, the wall-normal jet can be vectored to produce a pitched jet, similar to a VG jet. In addition, streamwise plasma actuators have been recently used to control turbulent boundary layer separation (Schatzman and Thomas 2010), control noise on a circular cylinder (Kozlov and Thomas 2009), modify supersonic flow (Im et al. 2010) and control laminar-turbulent transition (Hanson et al. 2010). Roy and Wang (2009) also demonstrated combined spanwise, streamwise and wall-normal flows by using DBD actuators with wavy structure.

We have already demonstrated that streamwise vortices can be generated by placing yawed DBD plasma actuators in a boundary layer. In this paper, we further the investigation of Okita et al. (2008) and study the mechanisms of streamwise vortex generation. We also explore parameters such as the actuator length, yaw angle, forcing magnitude and free-stream velocity on the streamwise vortex in a boundary layer over a flat plate. In addition, we demonstrate their potential for flow separation control by using DBD actuator arrays upstream of a ramp.

\section{Experimental setup and preliminary results}

An open return wind tunnel with 2-m-long test section with cross-section of $0.7 \mathrm{~m}$ by $0.25 \mathrm{~m}$ was used for this study. A ramp model, complete with endplates, was manufactured from 8 -mm-thick clear acrylic plate and placed at the start of the test section. The model was $1 \mathrm{~m}$ long and $0.284 \mathrm{~m}$ wide and had a super-elliptic shape leading edge with semimajor axis of $16 \mathrm{~mm}$, as shown in Fig. 1. DBD vortex generators were flush-mounted onto the upper surface of the model on a $0.2-\mathrm{m}$-long removable section, $0.5 \mathrm{~m}$ from the leading edge. The Reynolds number at the end of this removable section was in the range $90 \mathrm{k} \leq R e_{x} \leq 700 \mathrm{k}$. The boundary layer thickness was obtained from PIV data, which gave the value of $4.2 \leq \delta \leq 11.5 \mathrm{~mm}$ based on the height at which $U=0.99 U_{\infty}$. This was within $5 \%$ of the flat plate solution $\delta / x=5.0 \operatorname{Re}_{x}^{-1 / 2}$ (see White 1999). The shape factor was $H=2.49$ and the velocity profile closely matched the Blasius solution for a laminar flat plate boundary layer, as shown in Fig. 2.

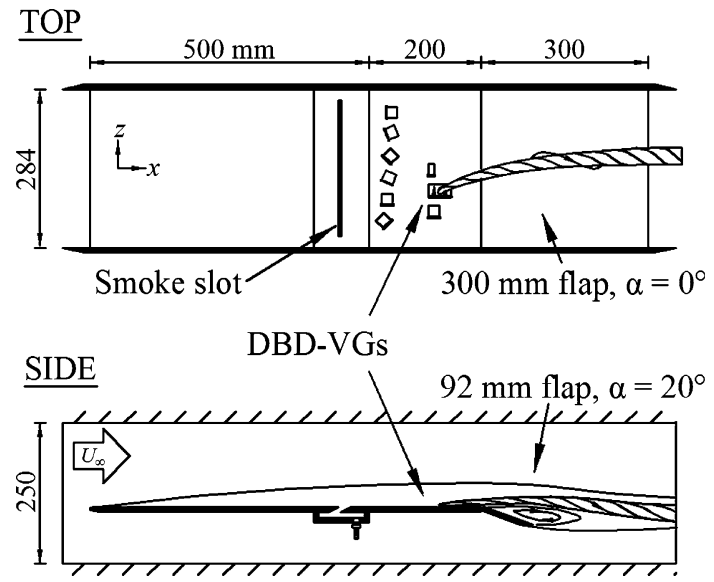

Fig. 1 Test model schematic. Smoke injection chamber shown below plate

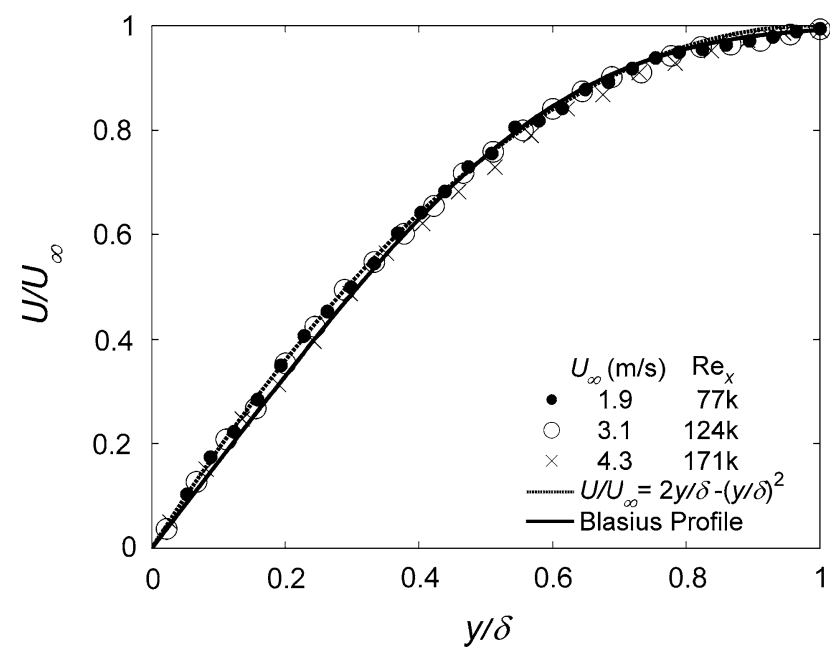

Fig. 2 Boundary layer profile measured with PIV at start of the DBDs, $605 \mathrm{~mm}$ downstream of the plate leading edge 
An adjustable flap was placed at the trailing edge of the model to create a ramp of variable angle. The flap was $300 \mathrm{~mm}$ long and set in the horizontal position in the first part of this paper, where we study the development of the DBD vortex generator flow. This created a 1-m-long smooth flat plate. In the latter part of this paper, we study the effect of the vortex generators on flow separation and a smaller 92-mm-long flap was installed and set at an angle of $20^{\circ}$ to the flat section. This simulated the trailing-edge region of an airfoil at high angles of attack, as studied in a similar configuration by Thompson and Whitelaw (1985). The gap between the two sections was smoothed with thin tape to produce a radius of approximately $40 \mathrm{~mm}$.

The DBD vortex generators (DBD-VGs) were constructed from $17-\mu \mathrm{m}$-thick copper electrodes separated by 250- $\mu \mathrm{m}$-thick Mylar dielectric. The upper electrodes were $2.5 \mathrm{~mm}$ wide, whilst the lower electrodes were $18 \mathrm{~mm}$ wide and placed so that the upper electrode leading edge was exactly at the lower electrode trailing edge (i.e. no overlap or gap, see Fig. 3). The actuator design was photochemically etched onto the substrate using standard PCB manufacturing techniques. High-voltage excitation was provided by a PSI-PG1040F power supply and the voltage and current was monitored on a Tektronix DPO4104 oscilloscope. A sinusoidally varying voltage signal was supplied to the electrodes with amplitude in the range $7 \leq E \leq 12 \mathrm{kV}_{\mathrm{p}-\mathrm{p}}$ and frequency $16 \leq f \leq 25 \mathrm{kHz}$. This produced glow discharge plasma which spread out to around $4 \mathrm{~mm}$ to the side of the upper electrode under which the lower electrode was placed (see Moreau 2007 and Corke et al. 2010 and references therein). This induced a flow vectored into the spanwise direction to produce the longitudinal vortex. The DBD-VGs were usually operated

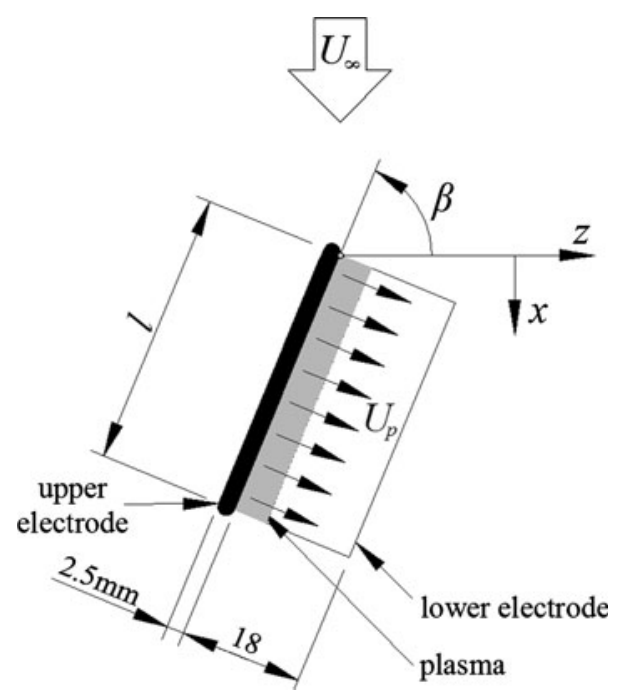

Fig. 3 Dielectric-barrier-discharge vortex generator (DBD-VG) schematic continuously for $1 \mathrm{~s}$, and flow field measurements were usually time-averaged for time $0.1 \leq t \leq 1$ s.

A Dantec 2D PIV system was used to measure the flow field in both the streamwise $(x-y)$ and cross-steam $(y-z)$ planes. The system consisted of a Litron LDY302-PIV 100 W Nd:YLF laser, a Vision Research Phantom V12.1 high-speed camera and a dedicated PC. Olive oil was used to seed the flow with nominally $1-\mu \mathrm{m}$ droplets using a rake in the settling chamber of the wind tunnel. A high-quality micro-screw-controlled 50-mm square mirror was carefully mounted at $45^{\circ}$ to the streamwise plane, $850 \mathrm{~mm}$ downstream of the flap trailing edge. This was deemed sufficiently far downstream to have no blockage effects on the upstream flow. For streamwise velocity measurement, the laser sheet was deflected upstream by the mirror, so that the light sheet illuminated the $x-y$ plane. The camera was then focussed through the acrylic wind tunnel side wall onto the appropriate measuring volume using a Sigma $105-\mathrm{mm}$ macro lens, set at $\mathrm{f} / 5$.6. For measurements in the cross-stream plane, the laser sheet was aimed through the wind tunnel wall to illuminate the $y-z$ plane whilst the camera viewed the measurement volume via the mirror. To minimise optical distortion and to maintain sufficient magnification, a Sigma 420-mm telephoto lens was used at f/8. Image pairs were typically taken at a frame rate of $200 \mathrm{~Hz}$, and PIV processing was performed using Dantec DynamicStudio v3.0. Velocity vectors were usually computed on a $16(y)$ by 32 ( $x$ or $z$ ) pixel grid with $50 \%$ overlap using a recursive cross-correlation technique (adaptive correlation with local median filter). This corresponded to an interrogation volume of typically $0.58 \times 1.16 \mathrm{~mm}$ for the cross-stream velocity components (or 14-40 vectors across $\delta$ ). This resolution was deemed sufficient to capture the streamwise vortex structure, where between 40 and 200 vectors were used in the integration for vortex circulation. In the majority of the vector figures presented in this article, we plot every other velocity vector in both $x$ (or $z$ ) and $y$ directions, but contour using the entire PIV field.

The general layout of the DBD-VG is shown in Fig. 3. We consider there to be four key parameters that will affect the streamwise vortex and its flow control capability: the plasma-induced velocity $U_{p}$, the free-stream velocity $U_{\infty}$, the yaw angle $\beta$ and the DBD-VG length $l$. The yaw angle is defined so that $\beta=0^{\circ}$ indicates purely streamwise forcing and $\beta=90^{\circ}$ indicates purely spanwise forcing. The coordinate axes are defined from the DBD-VG upstream tip. To study the effect of these parameters, DBD-VGs were manufactured with length $l=10,20$ and $40 \mathrm{~mm}$ and yaw angle $\beta=0^{\circ}, 22.5^{\circ}, 45^{\circ}, 67.5^{\circ}, 90^{\circ}$ and $135^{\circ}$ (drawn to scale in Fig. 1). The free-stream velocity was set in the range $2 \leq U_{\infty} \leq 15 \mathrm{~m} / \mathrm{s}$ with turbulence intensity $T_{i}<0.7 \%$ (measured with a hot-wire probe). The plasma velocity was set by changing the actuator voltage to 


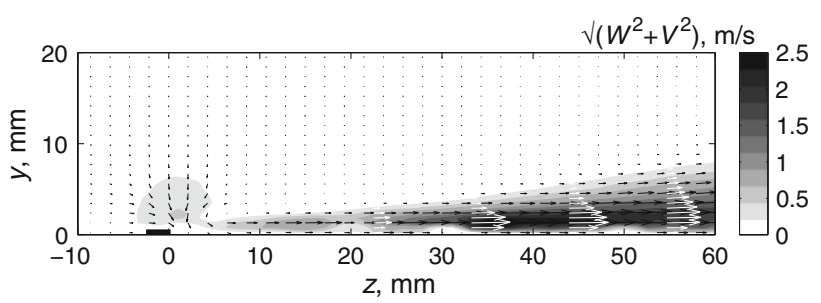

Fig. 4 Mean velocity field induced by a DBD-VG in quiescent air. $E=10 \mathrm{kV}_{\mathrm{p}-\mathrm{p}}, f=19.7 \mathrm{kHz}, \quad \beta=90^{\circ}, \quad l=40 \mathrm{~mm}, x=20 \mathrm{~mm}$, $U_{p}=2.2 \mathrm{~m} / \mathrm{s}, f_{p}=11.1 \mathrm{mN} / \mathrm{m}$

achieve $1 \leq U_{p} \leq 2.2 \mathrm{~m} / \mathrm{s}$. Here, we define $U_{p}$ from PIV measurements in quiescent air. Figure 4 shows a typical measurement, where the DBD produced a laminar wall jet directed away from the upper electrode. Due to erroneous PIV close to the wall and near the plasma, we define $U_{p}$ as the maximum jet velocity at $z=40 \mathrm{~mm}$. Although this is not equal to the velocity at the plasma itself, we feel that this is the best definition we can confidently and systematically measure with the experimental techniques available to us. Our PIV suggests that $U_{p}$ by this definition yields a velocity approximately two-thirds that which might be expected at the DBD origin. However, PIV is difficult in that region due to the large velocity gradients across the jet, laser reflections and the possibility that the seeding particles are influenced by the electric field. The momentum coefficient, $C_{\mu}=2 F_{p} / \rho U_{\infty}^{2} A$, was also measured where the plasma body force, $F_{p}$, was estimated from the momentum flux in quiescent air at $z=40 \mathrm{~mm}$, $F_{p}=\rho l \int W^{2} \mathrm{~d} y$, and $A$ is the plate planform area. In Fig. 4, $U_{p}=2.2 \mathrm{~m} / \mathrm{s}$, whilst $F_{p}=0.44 \mathrm{mN}$ (body force per unit width, $f_{p}=11.1 \mathrm{mN} / \mathrm{m}$ ). The momentum coefficient was in the range $0.001 \%<C_{\mu}<0.06 \%$. However, we will demonstrate later that it is the spanwise jet velocity that is the important scaling parameter for the generation of a streamwise vortex by DBD.

With flow, care was taken in measuring the cross-stream velocity distributions. To confidently measure these velocities, seeding particles must displace significantly in the $y-z$ plane before they drift through the laser sheet. This criterion becomes increasingly difficult as the free-stream velocity increases (strictly as the ratio of cross-stream to streamwise velocity reduces). In our data at $U_{\infty}=2 \mathrm{~m} / \mathrm{s}$, the maximum particle displacement was around 8-12 pixels within the streamwise vortex, but at $U_{\infty}=8 \mathrm{~m} / \mathrm{s}$, the displacement was only around 2-3 pixels. Increasing the time shift between image pairs, $\delta t$, could not solve this problem as the particles were then lost from the laser sheet. Thus, it was not possible to confidently measure $V$ and $W$ velocity components at high speed. In addition, the accuracy of the PIV decreased with distance from the centre of the image due to the "star field" effect as the particles move towards the camera. This was minimised somewhat by setting the vortex to be in the centre of the image and by using a long focal length camera lens to set a small angle of view. Furthermore, we time-averaged at least 150 vector maps for each crossstream velocity profile since the vortex was remarkably stable with time. A flow visualisation study was performed to compare with the PIV results. Here, smoke was injected through a thin slot $50 \mathrm{~mm}$ upstream of the DBD-VG test plate at an angle of $30^{\circ}$ to the wall, as shown in Fig. 1. The smoke injection system was only present for the flow visualisation study and was replaced with smooth acrylic in all other results. Note the addition of the injection system changed the blockage ratio in the wind tunnel from 3.7 to $6.5 \%$, but we do not expect the vortex structure to change significantly. Figure 5 shows a comparison between the PIV measurements and flow visualisation, where an instantaneous flow visualisation negative has been overlaid with time-averaged PIV a short distance downstream of a DBD vortex generator $(x / l=1.7)$. There is a very strong correlation between the flow visualisation and PIV measurements, which demonstrates confidence in our velocity measurement.

\section{Vortex formation mechanisms}

As shown in Fig. 5, it is clear that the DBD-VG can produce a concentrated streamwise vortex. After some initiation time of around $0.1 \mathrm{~s}$ (corresponding to the convection time $x / U_{\infty}$ ), the vortex was very stable and the core meandered by less than $\pm 2 \mathrm{~mm}$ whilst the plasma was activated. The flow field presented in Fig. 5 can therefore be considered as a steady-state phenomenon. When the plasma switched off, the vortex rapidly shrunk to the wall, taking less than $0.1 \mathrm{~s}$ to disappear completely. Thus, a longitudinal vortex structure can be turned on and off simply by intermittently energising the DBD.

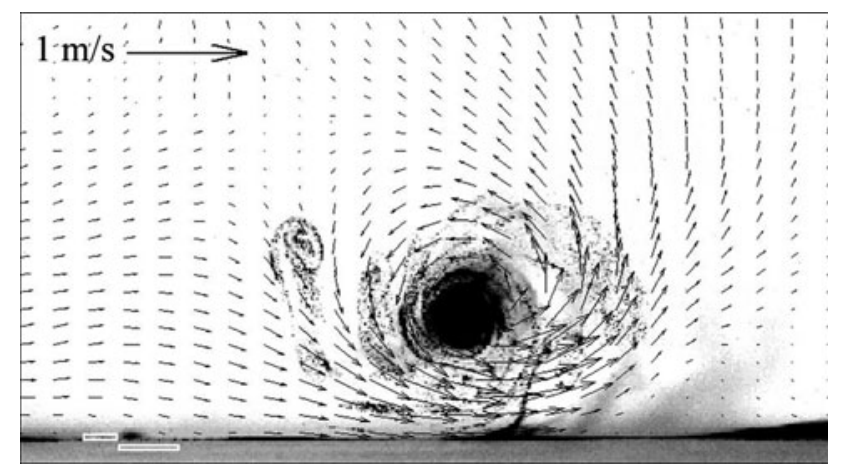

Fig. 5 Instantaneous flow visualisation negative overlaid with timeaveraged PIV in the $z-y$ plane. $\beta=90^{\circ}, l=40 \mathrm{~mm}, U_{\infty}=1.5 \mathrm{~m} / \mathrm{s}$, $U_{p}=1.05 \mathrm{~m} / \mathrm{s}, x=67.5 \mathrm{~mm}$. Field of view 69 by $39 \mathrm{~mm}$. Position of the DBD electrodes drawn at the lower left of the plot where the plasma is visible as the dark region in between the electrodes 
Fig. 6 Orthographic projection and $3 \mathrm{D}$ representation of streamwise vortex structure. Iso-surface marks local $\omega_{x} / \omega_{x, \max }=0.2$ with velocity magnitude and vectors at various cross-stream planes. $\beta=90^{\circ}, l=40 \mathrm{~mm}$,

$U_{\infty}=2.04 \mathrm{~m} / \mathrm{s}$,

$U_{p}=1.73 \mathrm{~m} / \mathrm{s}, U_{p} / U_{\infty}=0.85$. DBD plasma and upper electrode drawn in position for reference
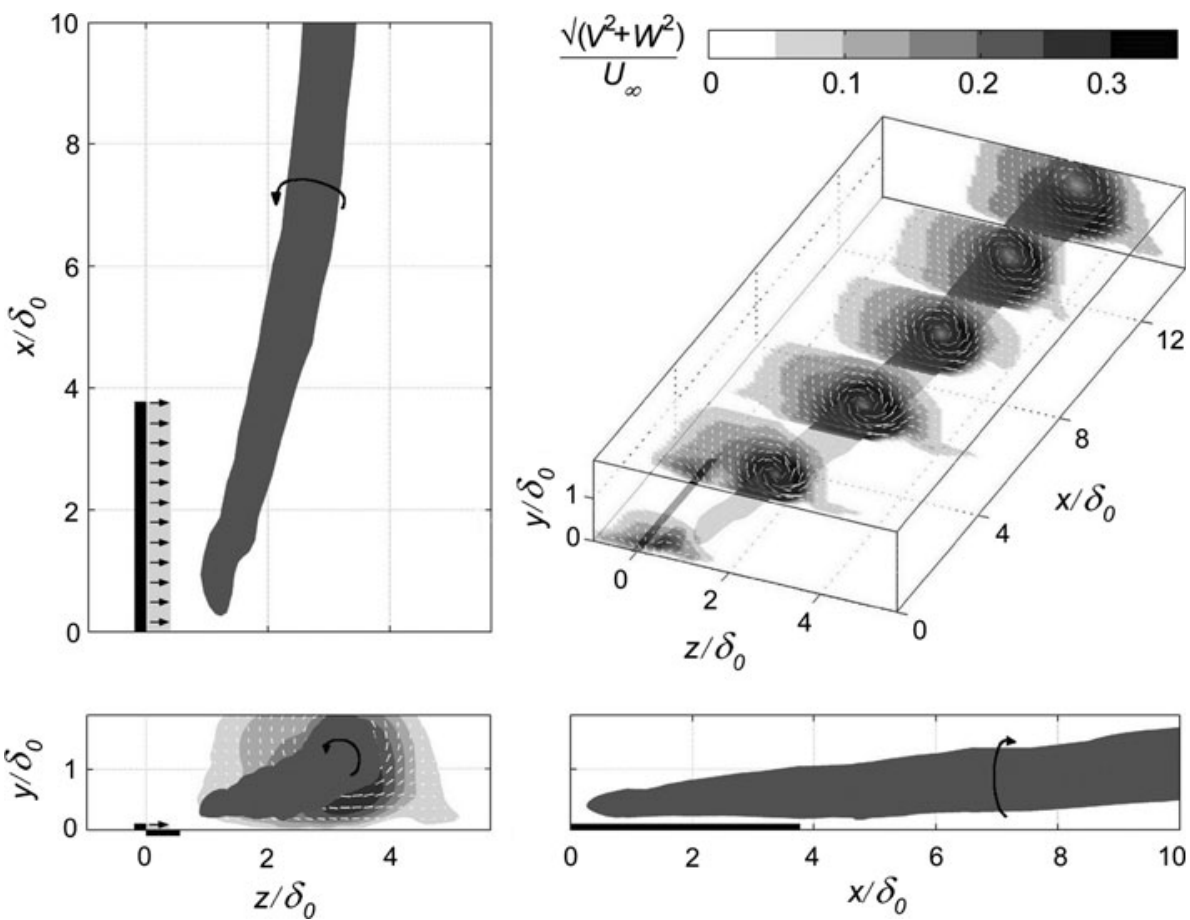

The streamwise vortex structure is shown in Fig. 6. In the three-dimensional representation, the cross-stream velocity magnitude and velocity vectors $(V, W)$ are plotted at various streamwise stations. In addition, the iso-surface that marks $20 \%$ of the peak vorticity within each plane is drawn orthographically to visualise the vortex. In this figure, the approaching laminar boundary layer flows in the positive $x$-direction, whilst the DBD-VG is located at $z=0$ in the region $0 \leq x / \delta_{0} \leq 3.8$ as drawn schematically on the plots $\left(0 \leq x \leq 40 \mathrm{~mm}, \delta_{0}\right.$ is the boundary layer thickness at the start of the DBD-VG). The DBD plasma produces a laminar wall jet in the positive $z$-direction only $\left(\beta=90^{\circ}\right)$, and it is clear that a well-defined streamwise vortex was created which increased in size as it developed downstream. Within the plasma region $\left(x / \delta_{0} \leq 3.8\right)$, momentum was continuously added to the vortex, so that the strength increased with distance downstream. Thereafter, the vortex spread out and decayed, although it was still readily discernable much further downstream than these plots at $x / \delta_{0}=28(x=300 \mathrm{~mm})$.

The mechanism of vortex formation is shown in Fig. 7, which plots streamwise vorticity contours at different stations. At the tip of the DBD-VG (frame a), the oncoming boundary layer fluid is primarily sucked towards the wall into the plasma $\left(0 \leq z / \delta_{0} \leq 0.3\right)$. It is then accelerated in the $z$-direction by the DBD body force to create a spanwise-directed jet close to the wall (frame $\left.\mathrm{b}, y / \delta_{0} \leq 0.2\right)$. The suction above the DBD is primarily due to mass continuity because the plasma imparts a body force to the fluid (i.e. the plasma is a source of momentum, not mass). This can also be clearly seen in the region directly above the DBD in quiescent air (Fig. 7g, which re-plots Fig. 4 for comparison). Therefore, fluid is drawn into the plasma from above it and to the side $(-z)$ to replace that accelerated in the $+z$-direction by the DBD body force. This action creates a circulation in the locality of the plasma actuator, which initiates the streamwise vortex above the wall jet. This process has some similarity to the formation of a starting vortex by DBD plasma in quiescent air (Jukes et al. 2008; Whalley and Choi 2010), although here the vortex is a steady structure that develops spatially rather than with time. However, the spanwise wall jet in this case should also become twisted into the streamwise direction by the oncoming flow. This means that the jet becomes increasingly vectored into the $x$-direction with distance from the electrode and distance downstream. The jet can be seen to lift away from the wall on the outside edge of the vortex (frame c, $z / \delta_{0} \approx 2$ ). Thus, the initially spanwise wall jet becomes twisted into $x$, lifted from the wall and then spirals around the vortex core. Within the planes of the DBD-VG (frames a-d), the plasma continually adds momentum to the vortex, so that it increases in strength as it develops. Downstream of the DBD-VG (frames e-f), the vortex slowly decays and translates laterally under its own self-induced velocity due to the vortex image in the wall. Note that the wall-ward suction in the region $0 \leq z / \delta_{0} \leq 0.3$ is not observed downstream of the DBD-VG.

The circulation around the streamwise vortex, $\Gamma$, was measured by integrating the streamwise vorticity over the 

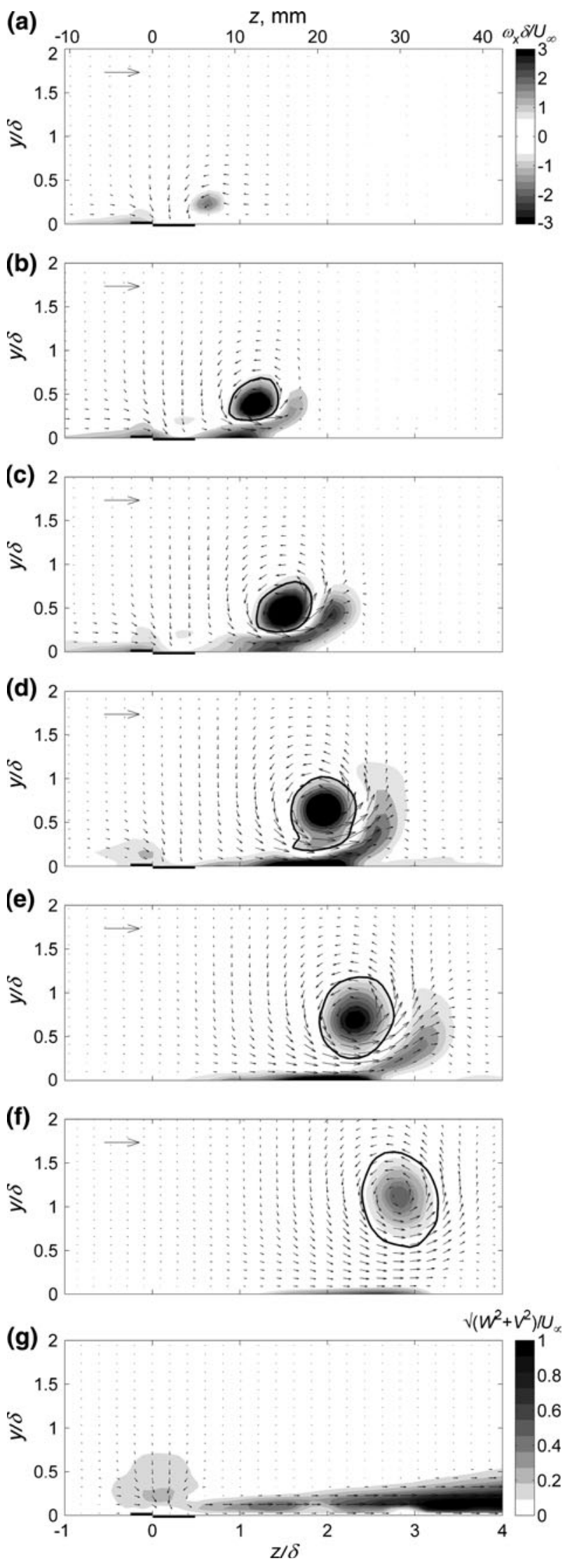

4 Fig. 7 a-f Vorticity magnitude and velocity vectors at $x / \delta_{0}=0,0.94$, $1.89,3.77,5.66,11.32(x=0,10,20,40,60,120 \mathrm{~mm})$; $\mathbf{g}$ velocity magnitude at $x / \delta_{0}=1.89$ in quiescent air. $\beta=90^{\circ}, l=40 \mathrm{~mm}$, $\delta_{0}=10.6 \mathrm{~mm}, U_{p} / U_{\infty}=0.85$. Reference vector shows $W=U_{\infty}$ and contours mark $+0.2 \omega_{x, \max }$. Cross-stream planes are downstream of the plasma region in e and $\mathbf{f}$. DBD electrodes drawn schematically in black

structure, determined by a $20 \%$ cut-off level of the peak vorticity (see iso-surface and contours in Figs. 6 and 7, respectively). In addition, the location of the vortex core $\left(y_{c}, z_{c}\right)$ was identified from the point of minimum velocity within this contour. We believe that the role of the DBDVG is to produce a wall jet in the spanwise direction which feeds the streamwise vorticity from below to increase its strength. Thus, the appropriate height and velocity scales of the vortex should relate to the thickness and spanwise velocity of the wall jet itself. Practically, however, these parameters are difficult to define because the wall jet evolves from the tip of the DBD-VG. This means that the thickness and velocity vary in all three spatial dimensions. Nevertheless, we test this scaling argument in Fig. 8. Here, the spanwise wall jet thickness, $\delta_{p}$, is defined as the height to one-half the maximum spanwise velocity directly underneath the vortex core ( $c f$. Fig. 7). This was measured at several stations along the DBD-VG $(0.25 \leq x / l \leq 1)$ and then linearly fit back to the DBD origin. It therefore indicates the hypothetical thickness of the plasma wall jet at its creation. The wall jet velocity, $W_{p}$, is defined as the maximum spanwise velocity directly underneath the vortex core towards the end to the VG. Since the jet takes some distance to establish, $W_{p}$ was averaged over the aft portion of the VG in the region $0.75 \leq x / l \leq 1$. This method yielded $W_{p}$ within $25 \%$ of $U_{p}$ (measured in quiescent air), although $W_{p}$ showed a weak dependence on the free-stream velocity. Figure 8 confirms that $\delta_{p}$ and $W_{p}$ scaling do

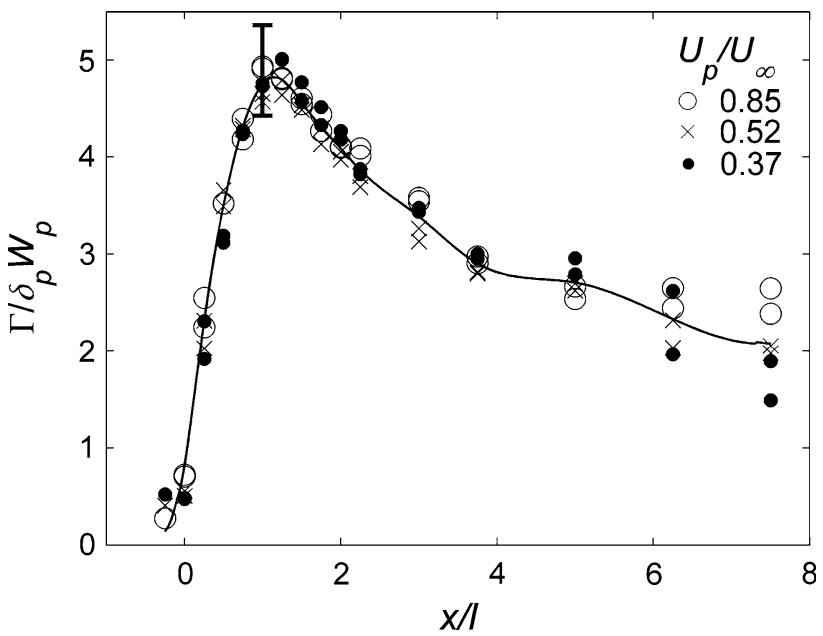

Fig. 8 Non-dimensional vortex circulation with distance downstream for various $U_{p} / U_{\infty} . \beta=90^{\circ}, l=40 \mathrm{~mm}, U_{p}=1.73 \mathrm{~m} / \mathrm{s}$ 
universally collapse the vortex circulation for a range of $U_{p} / U_{\infty}$. This demonstrates that $\delta_{p}$ and $W_{p}$ are the parameters involved in the formation of the streamwise vortex by DBD plasma. In contrast, Lögdberg et al. (2010) stated that the total circulation generated by vane-type vortex generators can be estimated as $\Gamma / h U_{\mathrm{VG}}=2 k$, where $h$ is the blade height, $U_{\mathrm{VG}}$ is the velocity at the blade tip and $k$ is a coefficient that is a function of vane geometry (typically $k=0.6)$. This demonstrates an important difference in the vortex formation mechanism between physical VGs and DBD-VGs. Here, the DBD-VG acquires circulation from the spanwise wall jet, whereas vane-type VGs acquire circulation from the twisting of the streamwise velocity. The experiment at $U_{p} / U_{\infty}=0.85, x / l=1$ was repeated eleven times with a range of PIV parameters to provide a measure of error (time between laser pulses, $\mathrm{d} T=225 \pm$ $125 \mu \mathrm{s})$. This is indicated by the $\pm 2 \sigma$ error bar from which the total error in $\Gamma$ is estimated to be $\pm 9 \%$ at $x / l=1$.

Figure 8 shows that the vortex circulation increased rapidly within the plasma region $(x / l \leq 1)$, where $\Gamma$ is generated at a rate of about $0.5 \mathrm{~m}^{2} / \mathrm{s}$ per-metre-DBD. This shows that the plasma adds circulation in an approximately linear manner along the DBD-VG length. The circulation reached a peak at the downstream edge of the DBD-VG and thereafter decayed. Pauley and Eaton (1988) discuss that a vortex can only loose circulation to skin friction at the wall, so that in the immediate downstream of the DBD, when the vortex lies close to the wall, the circulation loss is relatively rapid (approximately $0.1 \mathrm{~m}^{2} / \mathrm{s}$ per-metre-DBD in Fig. 8). It should be noted that this initial decay rate is significantly less than the growth rate. For $x / l>5$, however, the decay rate reduced indicating that the vortex is lifting from the surface. This can be seen in Fig. $7 \mathrm{f}$ where the core has lifted outside of the boundary layer, so that there is little interaction with the wall.

The spanwise and vertical locations of the vortex core $\left(z_{c}, y_{c}\right)$ are plotted in Fig. 9. Initially, the vortex moves rapidly away from the actuator in the spanwise direction. This lateral translation is less rapid in the downstream of the DBD and its motion is expected to be due to mutual induction with its image vortex in the wall (Whalley 2011). In contrast, the growth rate of the vortex in the $y$ direction is relatively constant, even downstream of the DBD. What is particularly interesting in Fig. 9 is the origin of the streamwise vortex. This suggests that the vortex originates to the side of the electrode, $z_{0} / \delta \approx 1.0$, slightly above the wall, $y_{0} / \delta \approx 0.3$, and at a short distance downstream of the tip, $x_{0} l l \approx 0.25$. As mentioned above, we believe that the primary role of the DBD-VG is to create a wall jet in the spanwise direction which becomes twisted into the streamwise direction by the oncoming boundary layer. Meanwhile a circulation is set up above the plasma by mass continuity. This initiates the vortex slightly downstream (a)
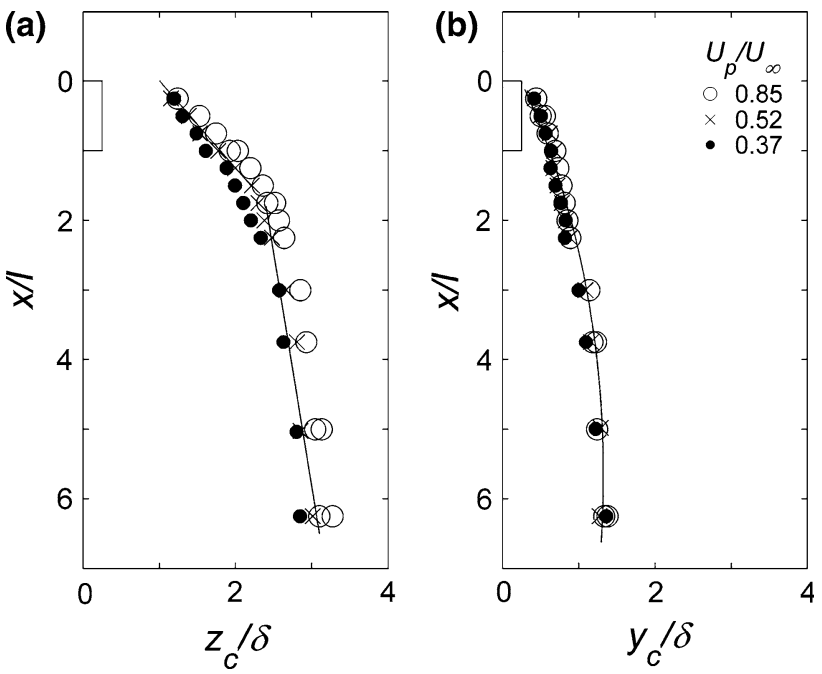

Fig. 9 Vortex core development for various $U_{p} / U_{\infty} . \beta=90^{\circ}, l=$ $40 \mathrm{~mm}$. Position of DBD-VG drawn schematically on the ordinate axis

and to the side of the DBD and a little way above the wall jet.

\section{Parameter optimisation of plasma VGs}

We now examine the effect of the velocity ratio $U_{p} / U_{\infty}$, actuator length $l$ and yaw angle $\beta$ on the streamwise vortex. Figure 10 shows the effect of $U_{p} / U_{\infty}$, where the vorticity and cross-stream velocity vectors at fixed plane $x / l=1.69$ $(x=67.5 \mathrm{~mm})$ are plotted for $0.3<U_{p} / U_{\infty}<1.2$. Here, the velocity ratio was changed through increasing $U_{\infty}$ only, but data for different $U_{p}$ will be presented later which show similar behaviour to that described here. Figure 10 shows that the vortex circulation and relative cross-stream velocity vectors decreased with decreasing $U_{p} / U_{\infty}$ (i.e. increasing $U_{\infty}$, frames a-e respectively), whilst the vortex size seems reasonably constant when scaled with $\delta$. However, in dimensional space, the vortex became much smaller as $U_{\infty}$ increased and the vortex forms much closer to the DBD-VG. Also, we found that the absolute magnitude of the spanwise jet velocity was reasonably constant with $U_{\infty}$, which suggests that the plasma produced a similar force, and hence induced a similar wall jet velocity, independent of $U_{\infty}$. However, the wall jet becomes twisted into the streamwise direction to a higher degree with increasing $U_{\infty}$, so that a smaller vortex forms closer to the electrode.

Figure 11 shows the non-dimensional vortex circulation with $U_{p} / U_{\infty}$. These measurements were taken at fixed $x / l=1.69$ downstream of the DBD-VG, so that $\delta_{p}$ and $W_{p}$ were interpolated back to the VG position assuming the same vortex development as in Fig. 8. Figure 11a shows a 

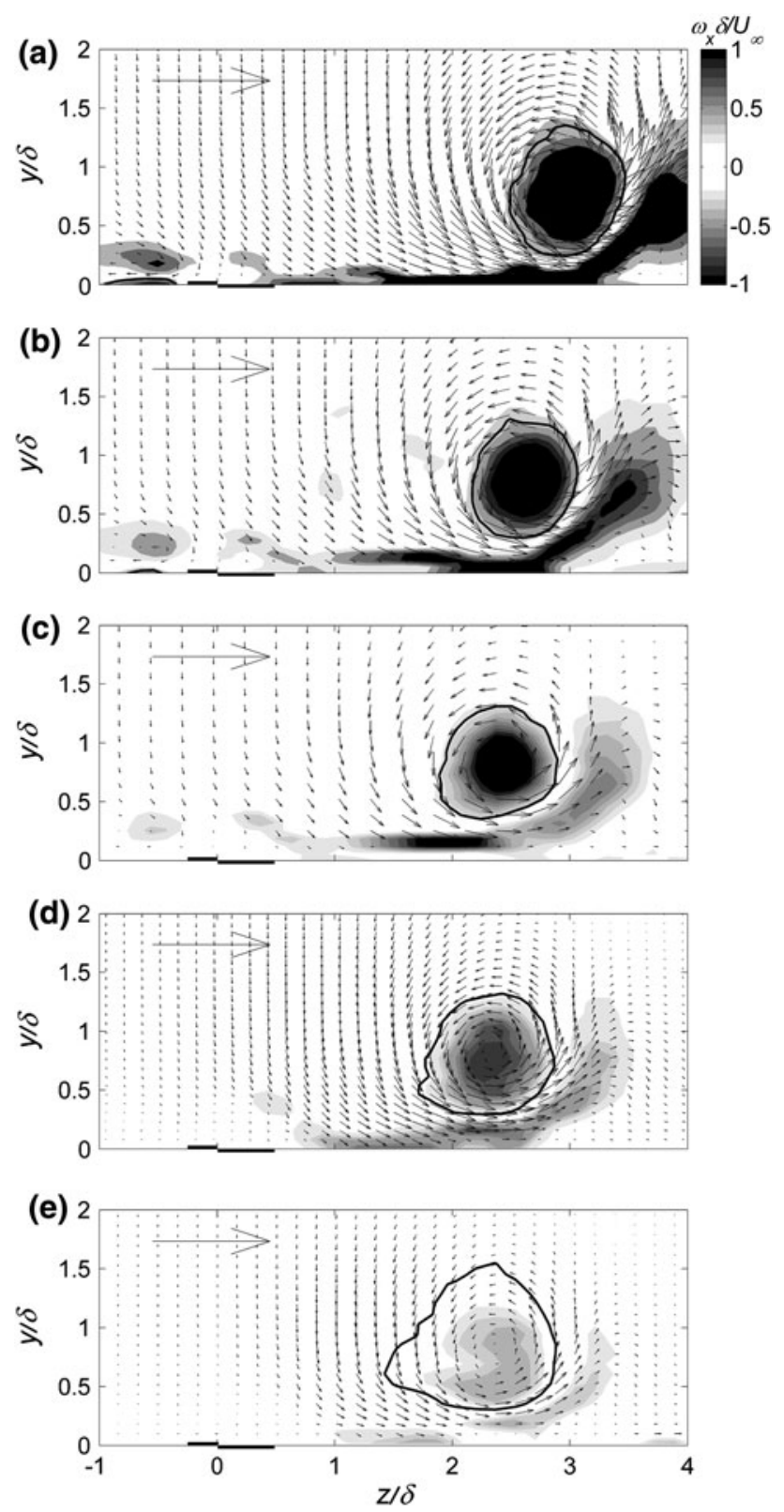

Fig. 10 Vorticity magnitude and velocity vectors at $U_{p} / U_{\infty}=1.17$, $0.73,0.52,0.39,0.32 . \beta=90^{\circ}, x / l=1.69, U_{p}=2.20 \mathrm{~m} / \mathrm{s}$. Reference vector shows $W=U_{\infty}$ and contour marks $+0.2 \omega_{x, \max }$ scatter of data around a value of $\Gamma / \delta_{p} W_{p}=4.3$ at low $U_{p} / U_{\infty}$. As $U_{p} / U_{\infty}$ reduces, the vortex becomes progressively smaller, so that the errors in our PIV measurements of $\Gamma, \delta_{p}$ and $W_{p}$ increase. Also, $\delta_{p}$ and $W_{p}$ may be influenced by the oncoming flow and not only by the plasma actuator (i.e. Reynolds number effects). However, in Fig. $11 b, \Gamma$ is scaled with the local undisturbed boundary layer thickness, $\delta$, and the free-stream velocity, $U_{\infty}$, so that the magnitude of the vortex strength can be compared at different $U_{p} / U_{\infty}$. This is similar to $h$ and $U_{\mathrm{VG}}$ scaling for a vane-type $\mathrm{VG}$, but we have used $\delta$ as the height scale since the DBD-VG does not protrude into the flow. Figure $11 \mathrm{~b}$ suggests that the vortex circulation varies as $\Gamma / \delta U_{\infty}=$ $1.1 U_{p} / U_{\infty}$, so that the strength linearly increases through increasing the plasma jet velocity. It is interesting here that the constant of proportionality is greater than 1 , which on face value suggests that more circulation can be created than is input by the plasma. This is, however, due to our underestimate of $U_{p}$ where earlier in Fig. 4, it was suggested that the velocity at the source of the plasma should be about $50 \%$ higher than measured by our definition. Thus, $\Gamma / \delta U_{\infty}=0.75 U_{p} / U_{\infty}$ or $\Gamma / \delta U_{p}=0.75$ is perhaps more realistic. Despite this, it is reassuring to see here that the levels of circulation achieved with the DBD-VG are comparable to those obtained by vane-type vortex generators $\left(\Gamma / h U_{\mathrm{VG}} \approx 1.2\right.$, see Lögdberg et al. 2010).

Next, we explore the effect of DBD-VG length. Figure 12 plots the vorticity maps for actuators with length $l=10,20$ and $40 \mathrm{~mm}$ at fixed cross-stream plane $x / l=2$. This measurement plane is downstream of the DBD-VGs, and it is clear that the longest DBD-VG generated the largest vortex since the plasma has imparted the greatest momentum into the flow. In addition, the lateral location of the vortex core increased with electrode length due to longer contact time with the DBD. The ac voltage and frequency driving each plasma discharge were fixed here ( $E=9.4 \mathrm{kV}_{\mathrm{p}-\mathrm{p}}, f=19.5 \mathrm{kHz}$ ), but we noticed a slight decrease in $U_{p}$ in quiescent air as $l$ decreased. We expect that this was because the plasma wall jet became increasingly three dimensional at small $l$ because end effects
Fig. 11 Vortex circulation with $U_{p} / U_{\infty} . \beta=90^{\circ}, l=40 \mathrm{~mm}$, $x / l=1.69$. a Scaling with $\delta_{p}$ and $W_{p}$ at $x / l=1.69$, line indicates $\Gamma / \delta_{p} W_{p}=4.3$. b Scaling with $\delta$ and $U_{\infty}$ at $x / l=1.69$, line indicates $\Gamma / \delta U_{\infty}=1.1 U_{p} / U_{\infty}$
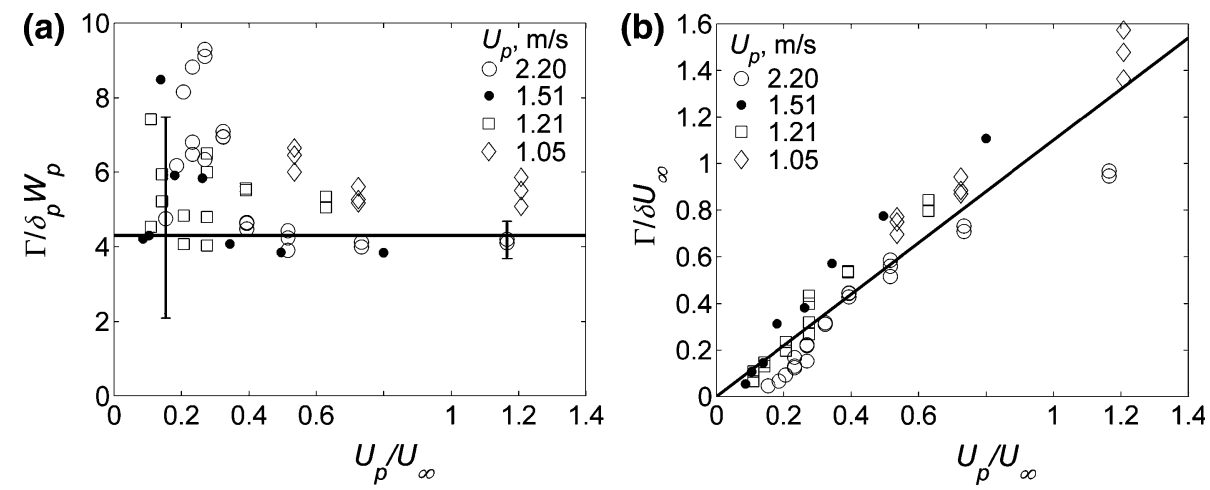

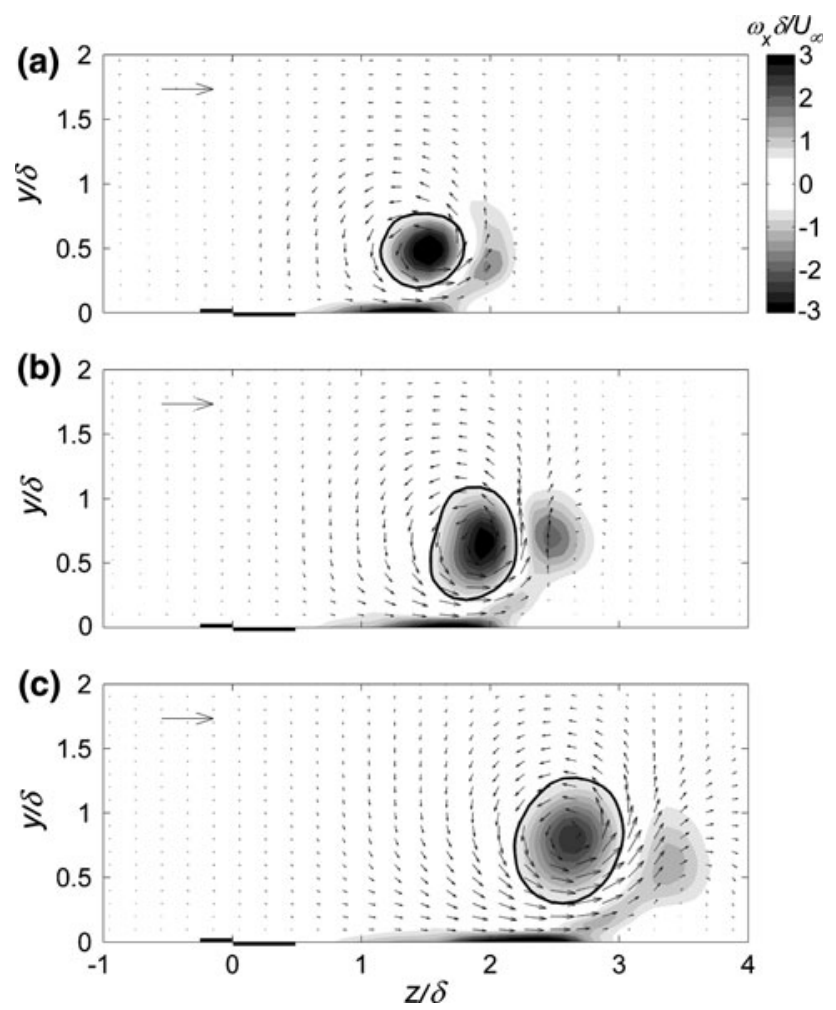

Fig. 12 Vorticity magnitude and velocity vectors for DBD-VG length, $l=10,20,40 \mathrm{~mm}$ in a-c, respectively. $x / l=2, \beta=90^{\circ}$, $U_{p} / U_{\infty}=0.64$. Reference vector shows $W=U_{\infty}$ and contour marks $+0.2 \omega_{x, \max }$

become increasingly important. We also observed that the power consumption per unit length increased slightly as $l$ decreased, suggesting that the electrical efficiency of the DBD was modified by the change in actuator geometry. The electrical characteristics of the DBD plasma actuator were not explored in detail in this study, but we believe that $U_{p}$ was approximately the same for each $l$ since $E$ and $f$ were not changed.

The vortex circulation is shown with streamwise distance in Fig. 13. Figure 13a shows self-similar profiles for each actuator, but different development for each $l$. This is because the vortex circulation did not reach a peak until beyond $x / l=1$ for short plasma VG actuators, which we believe is due to insufficient time for the spanwise wall jet to fully twist into the streamwise direction. Therefore, circulation increase does not stop until $x / l \approx 2$ and $x / l \approx 1.5$ for DBD-VGs of length $l=10$ and $20 \mathrm{~mm}$, respectively. However, for longer actuators, the circulation increase stops exactly at $x / l=1$.

Using scaling with $\delta$ and $U_{\infty}$ in Fig. 13b, the circulation appears to steadily increase within the plasma region at a similar same rate for all three DBD-VGs. Likewise, the circulation decays at a similar rate for each actuator. However, the peak differs with $l$. Thus, the plasma continually adds momentum into the vortex, which increases its circulation, but beyond the plasma region, the vortex starts to decay and loose energy to viscous interaction with the wall. Figure 13b therefore suggests that longer and stronger vortices can be formed merely by increasing the DBD length. However, one should expect that some limit will be reached for very long DBD-VGs because the energy added by the plasma should be balanced by the viscous losses at the wall. At this limit, the DBD can only act to maintain the vortex, rather than increase it. We did not reach this limit in these experiments, and our data suggest that significantly stronger vortices should be possible through simply increasing $l$.

In addition to the velocity ratio $U_{p} / U_{\infty}$ and the actuator length $l$, the streamwise vortex formation and strength should also depend on the DBD-VG yaw angle, $\beta$. Figure 14 plots vorticity maps at $x / l=1.5$ with increasing yaw angle in the range $22.5^{\circ} \leq \beta \leq 135^{\circ}$. It should be noted that $\beta=0^{\circ}$ corresponds to purely streamwise forcing, $\beta>90^{\circ}$ gives a component of force upstream into the oncoming flow and $z=0$ corresponds to the upstream tip of the plasma (see Fig. 3). The projected region of the DBD as seen from downstream has been indicated by the white box on the abscissa.

Figure 14 demonstrates that the vortex increases in size and strength with $\beta$ up to a limit at $\beta=90^{\circ}$ (frame d). At increased yaw angles, the vortex appears to be much weaker and more diffused. For example, at $\beta=135^{\circ}$ (frame e), we observed that the streamwise vortex was highly unstable. Possibly the oncoming boundary layer was
Fig. 13 Vortex circulation with downstream distance for DBDVGs of length, $l=10,20$, $40 \mathrm{~mm} . \beta=90^{\circ}$. a Scaling with $\delta_{p}$ and $W_{p}$ at $x / l=1.69$, $U_{p} / U_{\infty}=0.35-0.85$. b Scaling with local $\delta$ and $U_{\infty}$ $U_{p} / U_{\infty}=0.64$
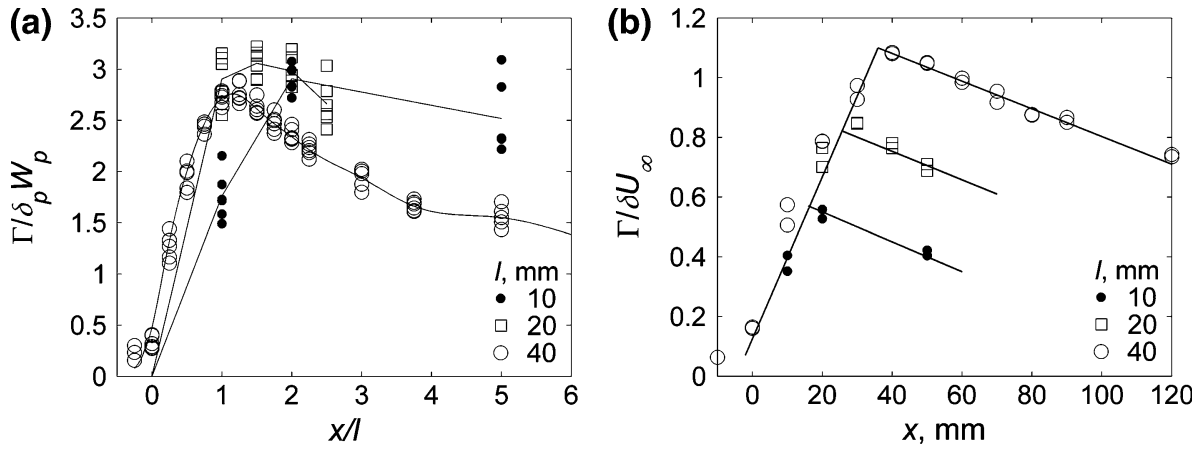

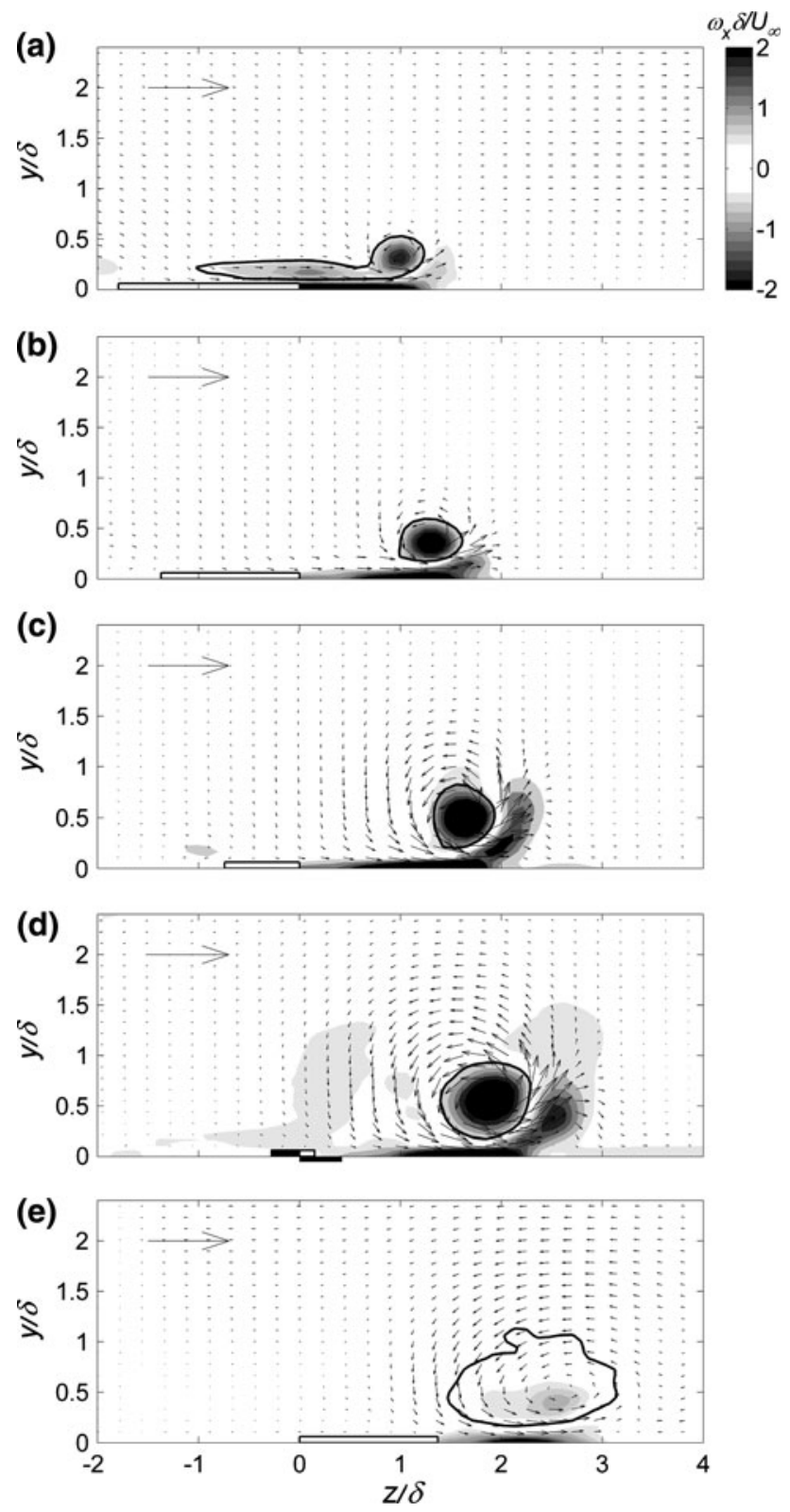

Fig. 14 Vorticity magnitude and velocity vectors for DBD-VG yaw angle, $\beta=22.5^{\circ}, 45^{\circ}, 67.5^{\circ}, 90^{\circ}, 135^{\circ}$ in a-e, respectively. $U_{p} / U_{\infty}=$ $0.65, x / l=1.5$. Reference vector shows $W=U_{\infty}$ and contour marks $+0.2 \omega_{x, \max }$. Projected region of the plasma drawn in white box separated by the upstream component of the wall jet, promoting laminar-turbulence transition. This case was not studied further since our experiment showed that an upstream-directed DBD would be quite unfavourable for generating a strong streamwise vortex. Figure 15 plots the circulation with $\beta$ at different $U_{p} / U_{\infty}$. Scaling with $\delta_{p}$ and $W_{p}$ in Fig. 15a collapses the data, again indicating that these parameters are the important scales for this flow. However, using scaling with $\delta$ and $U_{\infty}$ in Fig. 15b, a steady increase in circulation can be observed to a maximum at $\beta=90^{\circ}$ that drops off sharply thereafter. Thus, the optimum yaw angle to maximise the vortex strength is $\beta=90^{\circ}$.

\section{DBD vortex generator arrays}

Vortex generators for flow separation control are typically used in an array, so that a series of streamwise vortices are created along the surface span. To ensure effective flow control, the VGs must be placed sufficiently close together to ensure good spanwise coverage, yet far enough apart to avoid unfavourable interactions between adjacent vortices. This introduces a further geometrical parameter: the spanwise wavelength, $\lambda$. Furthermore, VGs can be arranged to produce either co-rotating (CoR) or counter-rotating (CtR) vortex pairs. In this section, we study two DBD vortex generator arrays designed to produce CoR and CtR vortices, and compare these structures with those from a single actuator.

We have demonstrated above that the circulation of the streamwise vortex created by a yawed DBD plasma actuator increases with $U_{p} / U_{\infty}$ and $l$ and reaches a maximum at $\beta=90^{\circ}$. Based on these results, DBD-VGs with yaw $\beta=90^{\circ}$ and length $l=40 \mathrm{~mm}$ were used in each array. The CoR array was a row of DBDs with similar geometry to that mentioned earlier (Fig. 3), placed at intervals of $\lambda=25 \mathrm{~mm}$. However, the lower electrodes were modified to be only $6 \mathrm{~mm}$ wide. This ensured that the lower electrode from one actuator was sufficiently far from the upper electrode of the adjacent actuator, so that no plasma formed
Fig. 15 Vortex circulation with DBD-VG yaw angle at different $U_{p} / U_{\infty} . x / l=1.5$. a Scaling with $\delta_{p}$ and $W_{p}$ at $x / l=1.5$. b Scaling with $\delta$ and $U_{\infty}$ at $x / l=1.5$
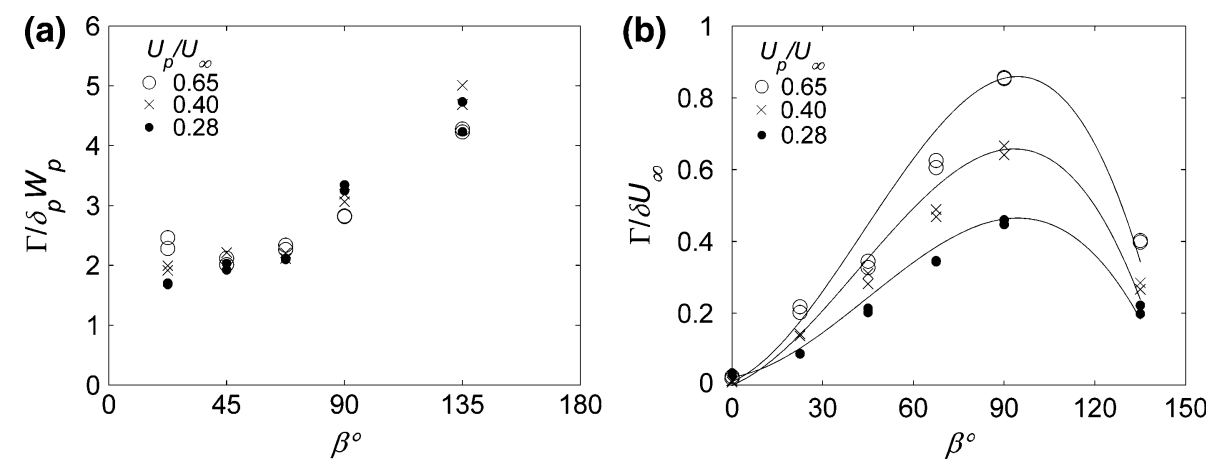

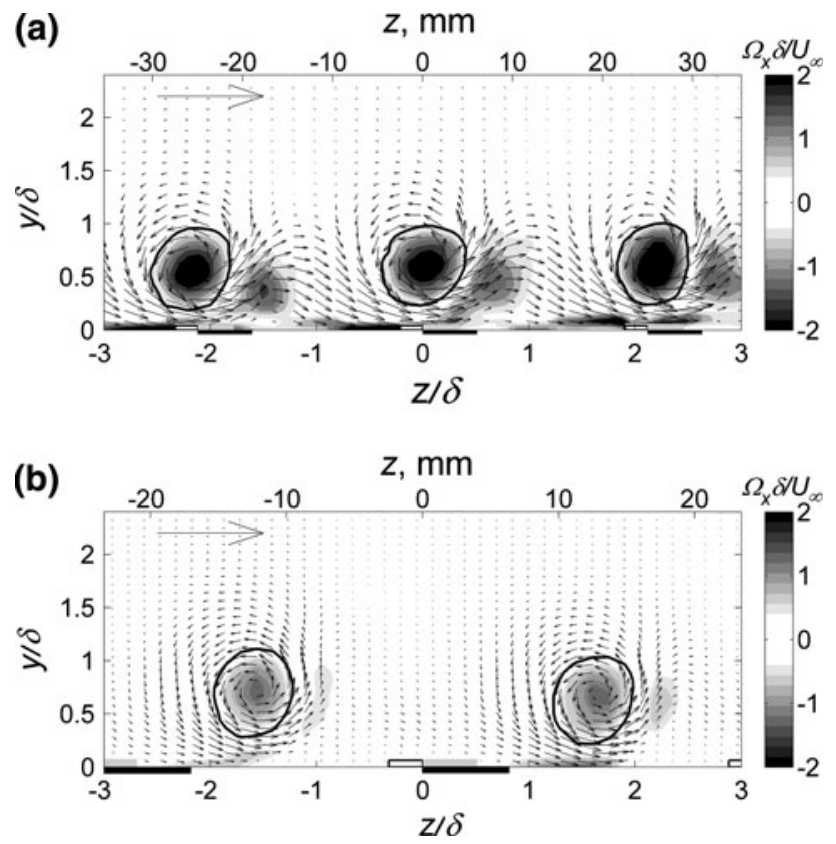

Fig. 16 Vorticity magnitude and velocity vectors for co-rotating DBD-VGs at a $U_{p} / U_{\infty}=0.63$ and $\mathbf{b} U_{p} / U_{\infty}=0.28 . x / l=1.63$, $\beta=90^{\circ}, \lambda=25 \mathrm{~mm}$. Reference vector shows $W=U_{\infty}$

on the rear side of it. We do not expect this modification to constrain the plasma on the front side since the discharge region was normally less than $4 \mathrm{~mm}$ wide. The CtR array had upper electrodes placed with $\lambda=50 \mathrm{~mm}$ and a lower electrode that covered the entire span of the actuator sheet. This meant that plasma formed equally to both sides of the upper electrodes to produce bi-directional wall jets, as described by Jukes et al. (2006). It should be noted that although $\lambda$ was different for each array, the same number of vortices was produced per unit width since two vortices were created by each CtR actuator. Each array spanned the test plate and there were 8 and 4 actuators in the CoR and CtR arrays, respectively. These actuators had to be used at reduced voltage compared to the earlier work ( $E=8.2 \mathrm{kV}_{\mathrm{p}-\mathrm{p}}$ and $f=14.4 \mathrm{kHz}$ ), because several actuators were energised at the same time which saturated our electrical supply at higher voltages due to power limitations. The wall jet from a single DBD-VG in quiescent air had velocity $U_{p}=1.21 \mathrm{~m} / \mathrm{s}$ at this voltage and frequency.

Figure 16 shows the induced flow by the CoR DBD vortex generator array at $x / l=1.63$ at two different velocity ratios. The DBD electrodes and the regions of plasma discharge have been drawn schematically on the abscissa. The figure clearly shows a system of co-rotating vortices. At $U_{p} / U_{\infty}=$ 0.63 (frame a), there is much interaction between adjacent vortices, whilst at $U_{p} / U_{\infty}=0.28$ (frame b), the vortices are effectively independent with characteristics as described above. Figure 17 compares the circulation produced with this array to a single DBD-VG. The circulation of each vortex was

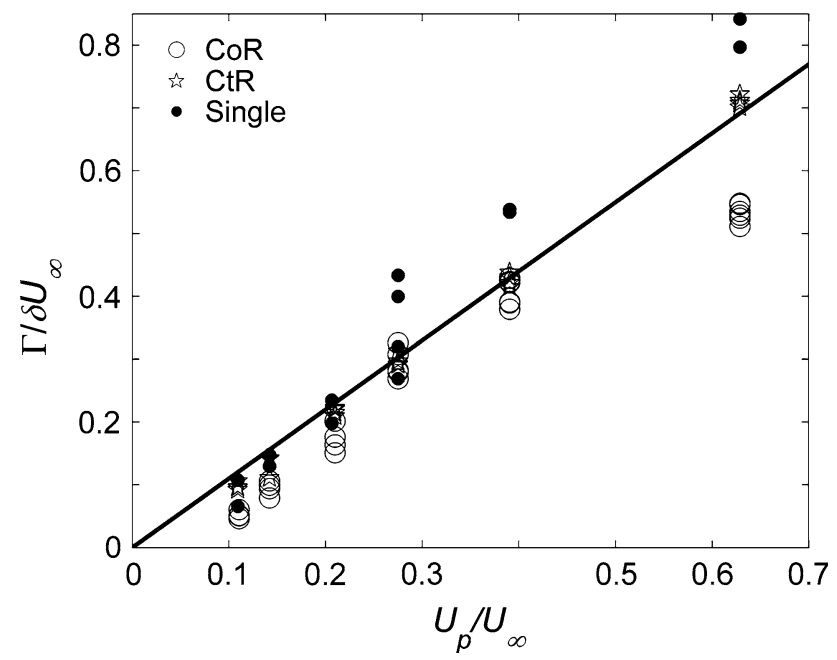

Fig. 17 Vortex strength with $U_{p} / U_{\infty}$ for CoR and CtR arrays, and a single DBD-VG. $x / l=1.63, \beta=90^{\circ}$
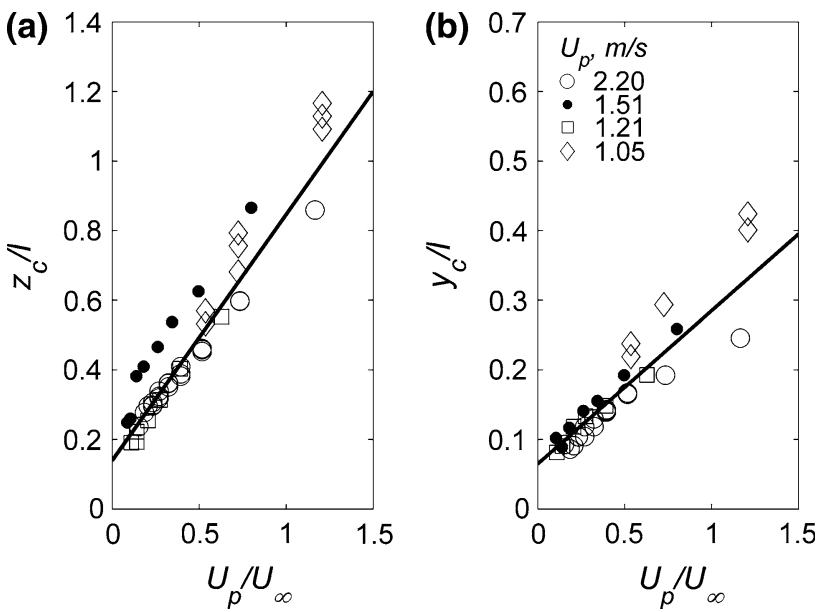

Fig. 18 Vortex core location with $U_{p} / U_{\infty}$ for a single DBD-VG. $x / l=1.69, \beta=90^{\circ}$

less for the CoR array, particularly at $U_{p} / U_{\infty}=0.63$ where $\Gamma / \delta U_{\infty}$ has decreased by more than $30 \%$. This seems to be due to the vortex-vortex interactions as the spanwise spacing was too small. This has also been observed in vane-type VGs (Pearcey 1961) and is an inefficient configuration for flow control since some of the useful circulation is mutually lost. Whether the vortices interact or not for the DBD-VGs should therefore depend on both $U_{p} / U_{\infty}$ and $\lambda$. Figure 18 shows the location of the core from a single actuator with $U_{p} / U_{\infty}$. Using these data, $\lambda$ should be chosen according to the intended $U_{p} / U_{\infty}$ of the application. For example, $\lambda$ might be chosen to be sufficiently greater than $z_{c}$ (using the linear fit in Fig. 18a, $\lambda l \approx 2 z_{c} l=1.4 U_{p} / U_{\infty}+0.3$ can be recommended). Of course, the vortices will eventually meet downstream, but this criterion should prevent interactions until after the vortices have been useful for flow control. The optimal spacing for flow separation control has been discussed by several authors, 

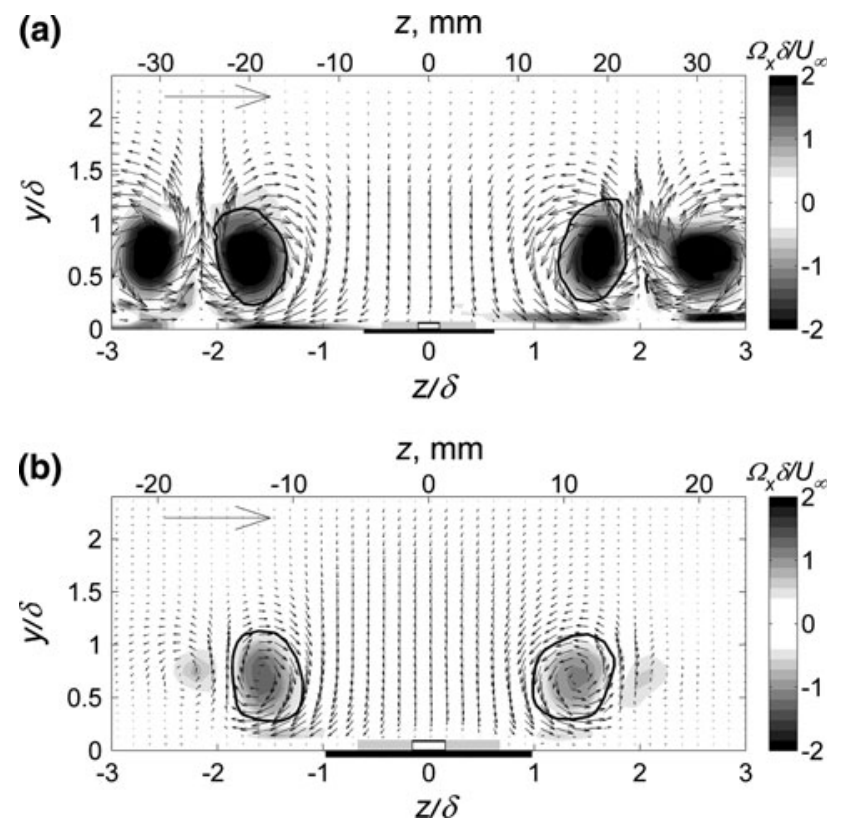

Fig. 19 Vorticity magnitude and velocity vectors for counter-rotating DBD-VGs at a $U_{p} / U_{\infty}=0.63$ and $\mathbf{b} U_{p} / U_{\infty}=0.28 . x / l=1.63$, $\beta=90^{\circ}, \lambda=50 \mathrm{~mm}$. Reference vector shows $W=U_{\infty}$

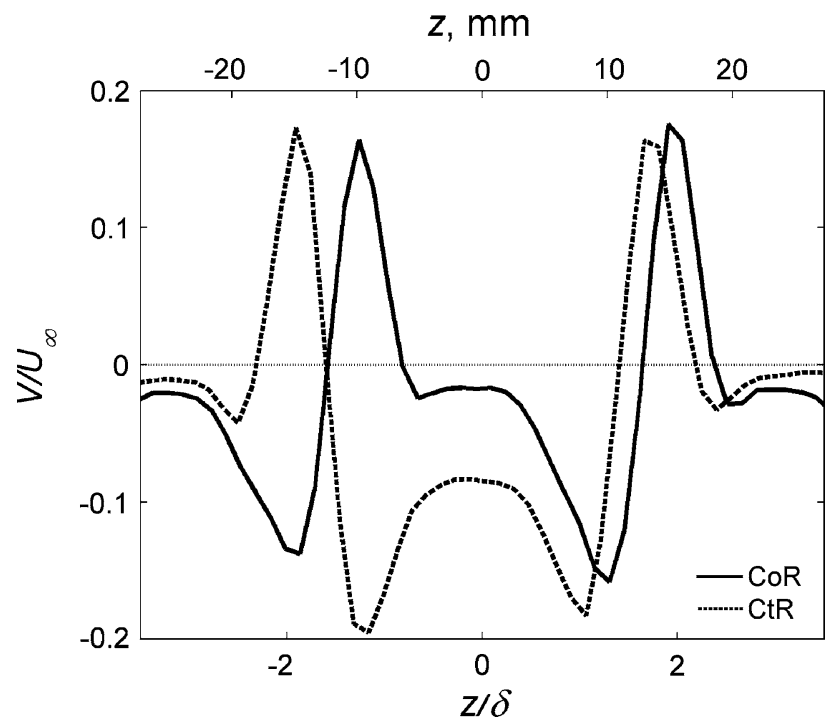

Fig. 20 Wall-normal velocity distribution at the vortex cores for CoR and CtR DBD-VGs. $x / l=1.63, \beta=90^{\circ}, U_{p} / U_{\infty}=0.28$

notably in the study by Godard and Stanislas (2006a). For CoR vane-type VGs, they found an optimal spacing $\lambda / h=6$ with height $h / \delta=0.37$. In a laminar boundary layer at the $\mathrm{VG}$ tip $U_{\mathrm{VG}} / U_{\infty}=0.60$ (see Fig. 2), so assuming $U_{\mathrm{VG}}$ is equivalent to $U_{p}$, our above criterion yields $\lambda l=1.14$. For the data in Fig. 18, $3.5 \leq l / \delta \leq 8.0$, which means that our suggested spacing is equivalent to $4 \leq \lambda / h \leq 9$, similar to their study.

Figure 19 shows the flow induced by the CtR vortex generator array. Again the actuator and plasma region have been drawn at the bottom of the plot. Each DBD actuator produced bi-directional plasma that forms a jet in both directions away from the upper electrode. This created a counter-rotating vortex pair that has common-flow down in the region directly above the plasma. At $U_{p} / U_{\infty}=0.63$ (a), each vortex in the vortex pair interacts with the vortices from the adjacent actuators, so that at this measurement location vortex pairs are created with common-flow up between them. In contrast at $U_{p} / U_{\infty}=0.28$ (b), there is no interaction with adjacent vortex pairs, so that only a common-flow down vortex pair is formed. Again this emphasises the importance of correct choice of $\lambda$, which depends on $U_{p} / U_{\infty}$. However, the interaction between adjacent CtR vortices is not as severe as the CoR case. Returning to Fig. 17, the strength of each vortex in the CtR pair is a better match to the single DBD-VG case than the CoR array. This is because adjacent vortices at this stage in their development combine, so that they do not counteract each other and their interactions are less destructive.

Figure 20 compares the wall-normal velocity across the vortex cores for $\mathrm{CoR}$ and $\mathrm{CtR}$ cases at $U_{p} / U_{\infty}=0.28$. The location of the left-hand vortices exactly coincides $\left(z_{d} \delta=-1.6\right)$, whereas there is a small difference in the locations of the right-hand vortices $\left(z_{d} \delta \delta=1.6,1.4\right.$ for CoR and CtR, see Figs. 16b and 19b). Nevertheless, the maximum velocity away from the wall $(+V)$ is nearly the same in both arrays, whilst the velocity towards the wall is significantly greater for the CtR case. This demonstrates that the CtR vortices effectively work together to create additional downwash, which is enhanced by the entrainment towards the plasma actuators.

These observations compare well with other studies on streamwise vortices created by vane-type VGs and VG jets. For example, the cross-stream velocity map presented in Fig. 19a is very similar to that presented in Godard and Stanislas (2006a) for an optimal counter-rotating array of triangular vanes. The size of the vortices differs due to different experimental facilities, notably $\delta$, but the qualitative image of the counter-rotating vortices is nearly identical. Furthermore, the DBD-VGs also compare well to single vortex and CoR arrays produced by inclined jets by Zhang (2000, 2003). However, the primary difference between the DBD-VG and these other methods is that the DBD-VG also induces a flow towards the wall directly above the plasma region. This suction is due to continuity since the plasma is a source of momentum, not fluid, as discussed above.

\section{Flow separation control}

The flow separation control ability of DBD vortex generators is now demonstrated over a ramp. This was set at an 

streamwise and spanwise slices in the region of the deflected $U_{p} / U_{\infty}=0.53\left(U_{p}=1.03 \mathrm{~m} / \mathrm{s}\right.$, $\left.U_{\infty}=1.96 \mathrm{~m} / \mathrm{s}\right), l=40 \mathrm{~mm}$, $\beta=90^{\circ}, \lambda=25 \mathrm{~mm}$. Figure stretched by a factor of 3 in the $z$ axis to aid viewing
Fig. 21 Velocity magnitude in flap with co-rotating DBD-VGs.
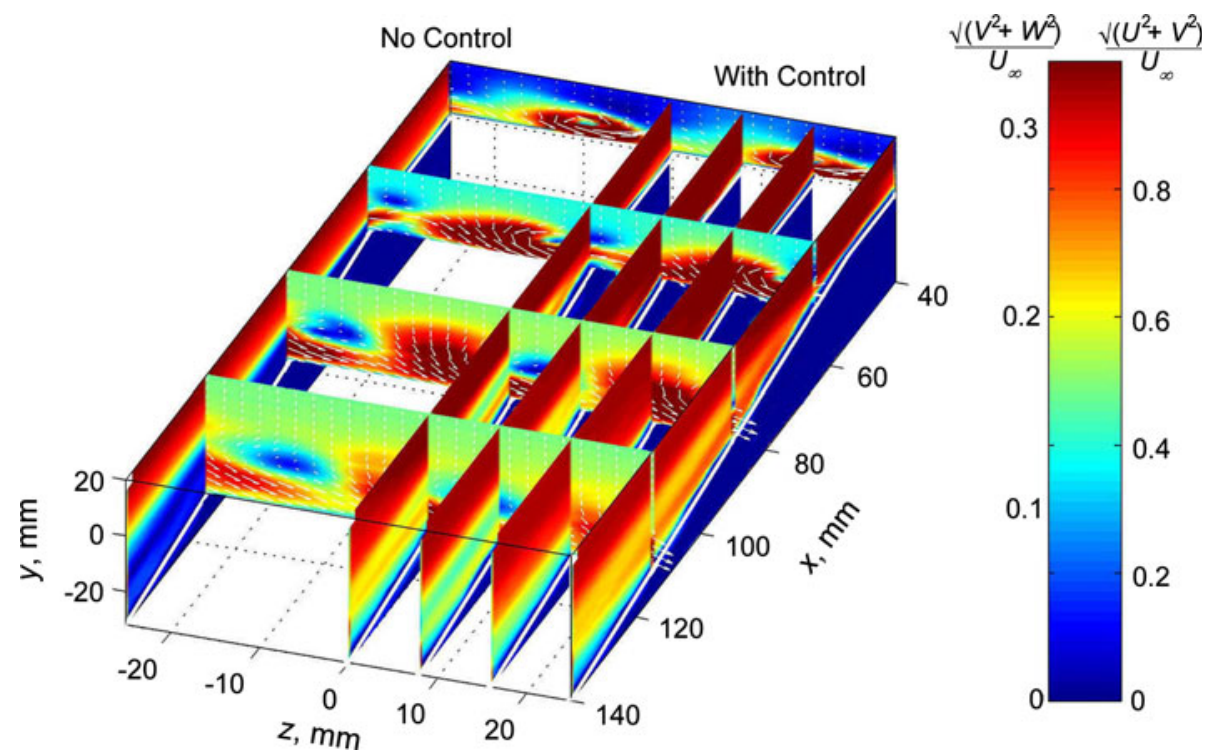

Fig. 22 Velocity magnitude in streamwise and spanwise slices in the region of the deflected flap with counter-rotating DBD-VGs. $U_{p} / U_{\infty}=0.53$, $l=40 \mathrm{~mm}, \beta=90^{\circ}$,

$\lambda=50 \mathrm{~mm}$. Figure stretched by a factor of 3 in the $z$ axis to aid viewing

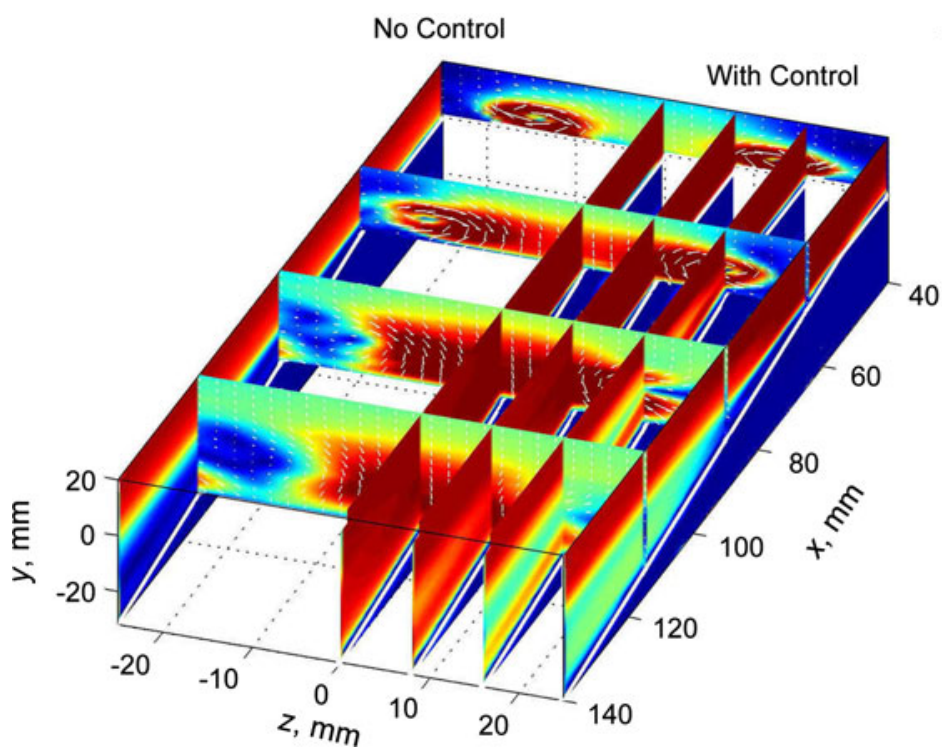

angle of $20^{\circ}$ to the flat plate to simulate the trailing-edge region of an airfoil at high angles of attack, see Fig. 1. The same CoR and CtR arrays discussed in the previous section were placed a short distance upstream of the ramp. As before, $x=0$ corresponds to the leading edge of the DBDVGs (placed $645 \mathrm{~mm}$ from the leading edge of the flat plate), and the actuators had length $l=40 \mathrm{~mm}$. The ramp started at $x=55 \mathrm{~mm}$ and finished at $x=141 \mathrm{~mm}$.

Figures 21 and 22 present time-averaged streamwise and cross-stream velocity slices for the CoR and CtR DBD vortex generator arrays, respectively. In both cases, the streamwise velocity profile without plasma is shown on the left-hand side for reference $(z=-25 \mathrm{~mm})$. This shows a large region of low-speed recirculating fluid above the ramp, indicating that the flow was completely separated. The flow with DBD-VGs is shown in all other slices. Note that the streamwise and cross-stream planes have been plotted with slightly different colour maps $\left(\sqrt{ }\left(U^{2}+V^{2}\right)=3 \sqrt{ }\left(V^{2}+W^{2}\right)\right)$. Velocity vectors have been plotted on the cross-stream planes only, and the figure has been stretched by a factor of three in the $z$-direction to aid viewing.

Figures 21 and 22 clearly show that CoR and CtR DBDVGs were both successful in controlling the flow separation. The velocity near the ramp surface has increased significantly in both cases, and the recirculation region appears narrower and reduced. For the CoR case (Fig. 21), the array of streamwise vortices persists right to the end of the ramp. They move laterally as they travel downstream (i.e. to the right in the figure). For example, the vortex core on the left-hand side travels from approximately $z=-10 \mathrm{~mm}$ at the start of the ramp to around $z=10 \mathrm{~mm}$ $(\Delta z / \lambda=0.8)$ towards the end of the ramp. On the left-hand 
side of each vortex in the plane of Fig. 21, there are large regions of induced downwash. This brings high momentum fluid towards the wall and reattaches the flow. Interestingly, and unlike the attached vortices in Fig. 16, there does not appear to be significant upwash on the right-hand side of these vortices. It should be noted that the flow does not totally reattach over the ramp, but the streamwise velocity maps are, however, reasonably uniform across the span. This is because the downwash regions are swept laterally due to the curved trajectory of the vortices, which leads to reasonably homogeneous flow control.

In contrast, the vortex pairs generated in the CtR case (Fig. 22) did not undergo lateral displacement and remain at roughly the same spanwise location along the ramp $(z \approx \pm 15 \mathrm{~mm}, z / \lambda \approx \pm 0.3)$. The vortices grow as the travel downstream, but they do not meander like the CoR case. There is a much larger region of downwash due to the combined action of the vortex pair. This acts to completely reattach the flow for $-10<z<10 \mathrm{~mm}(\mid z / / \lambda=0.4)$. However, flow separation control was not so successful outside of this region. For example at $z=25 \mathrm{~mm}(z / \lambda=0.5)$, there is a clear wake, but this is still significantly reduced compared to the flow without plasma. This region does not, however, have as reduced a flow separation as the CoR case at the same location. Therefore, the CtR array might be more effective at flow separation control directly downstream of the DBD, but it is less homogeneous across the span.

Figure 23 shows the velocity magnitude and vectors at $z=0 \mathrm{~mm}$, without and with CtR and CoR DBD-VGs at $U_{p} / U_{\infty}=0.53\left(U_{\infty}=1.96 \mathrm{~m} / \mathrm{s}\right)$. As above, it is clear that the DBD-VGs vastly reduced the flow separation region and they were very successful at reattaching the flow. For the CtR case (Fig. 23c), the flow has completely reattached, and all the velocity vectors run parallel to the plate. A thin boundary layer can be seen on the surface that separates only at the trailing edge. Meanwhile, the flow has not completely reattached for the CoR case but there is a large reduction in the wake, (Fig. 23b). Here, the velocity vectors show flow away from the wall at the start of the ramp and a band of low velocity above the ramp for $80<x<120 \mathrm{~mm}, y \approx 0 \mathrm{~mm}$. This is caused by the passage of the streamwise vortices through this plane. With reference to Fig. 21, CoR vortices bring high momentum fluid from their left to the region underneath, resulting in this low velocity band as the vortex core moves through the plane of Fig. 23b. The flow away from the wall at the start of the ramp is because the vortex is initially in front of this plane, so that its rotation lifts fluid away from the wall.

Figure 24 shows the same configuration but for much reduced $U_{p} / U_{\infty}=0.07\left(U_{\infty}=14.9 \mathrm{~m} / \mathrm{s}\right)$. The separation region without plasma is naturally thinner at this freestream velocity as shown in Fig. 24a. Comparison with Fig. 23 shows the flow control ability of the DBD-VGs
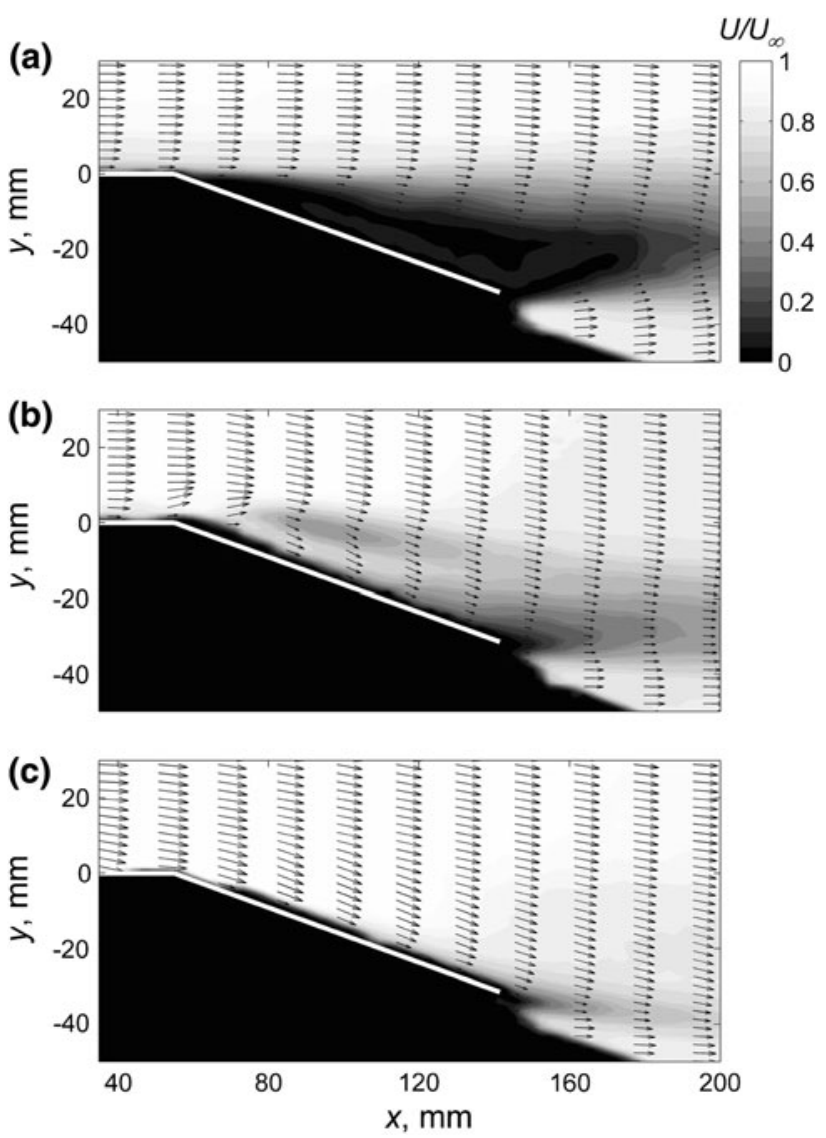

Fig. 23 Streamwise velocity magnitude a without plasma, b with CoR DBD-VGs and c with CtR DBD-VGs. $U_{p} / U_{\infty}=0.53$, $l=40 \mathrm{~mm}, \beta=90^{\circ}, z=0 \mathrm{~mm}$

reduced as $U_{p} / U_{\infty}$ reduced. With CoR DBD-VGs (Fig. 24b), there was very little modification to the separation region. The wake was slightly thinned but we can consider here that the plasma is simply too weak for effective flow control. For the CtR case, however, (Fig. 24c), the wake has still been affected by the vortex generators. The wake is obviously thinned and flow separation has been partially avoided. It is remarkable here that flow control was still possible with a plasma-to-free-stream velocity ratio of less than $10 \%$.

We quantify the flow control ability of plasma actuator arrays in Fig. 25 by showing the momentum flux across the wake at $x=180 \mathrm{~mm}$ with and without DBD-VGs. The increase in the wake momentum flux in this figure suggests that there is a reduction in drag due to the modification of the flow separation region by DBD-VGs. The data show larger variation in the spanwise direction with CtR DBDVGs, due to the increased spanwise inhomogeneity, but on average the CtR array was more effective than the CoR array. The effectiveness of flow control increases with $U_{p} / U_{\infty}$, where perhaps $U_{p} / U_{\infty}$ should be at least $20 \%$ in practical applications. 

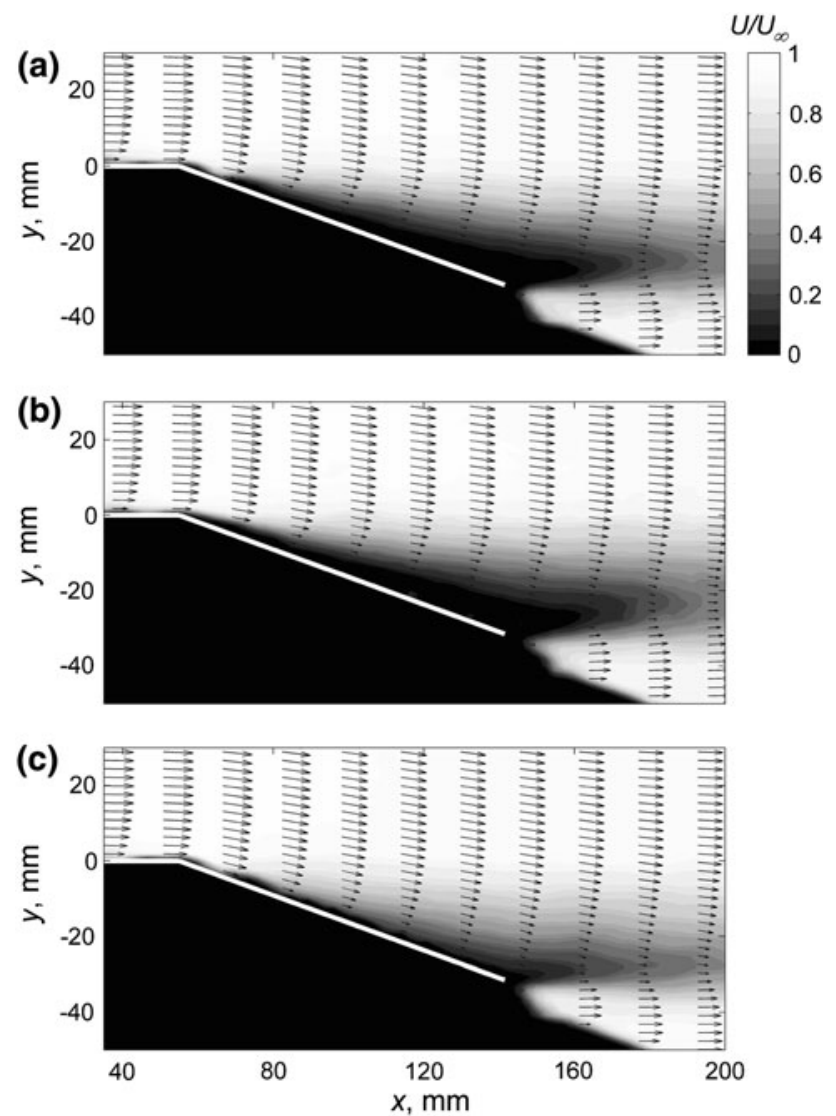

Fig. 24 Streamwise velocity magnitude a without plasma, b with CoR DBD-VGs and c with CtR DBD-VGs. $U_{p} / U_{\infty}=0.07$, $l=40 \mathrm{~mm}, \beta=90^{\circ}, z=0 \mathrm{~mm}$

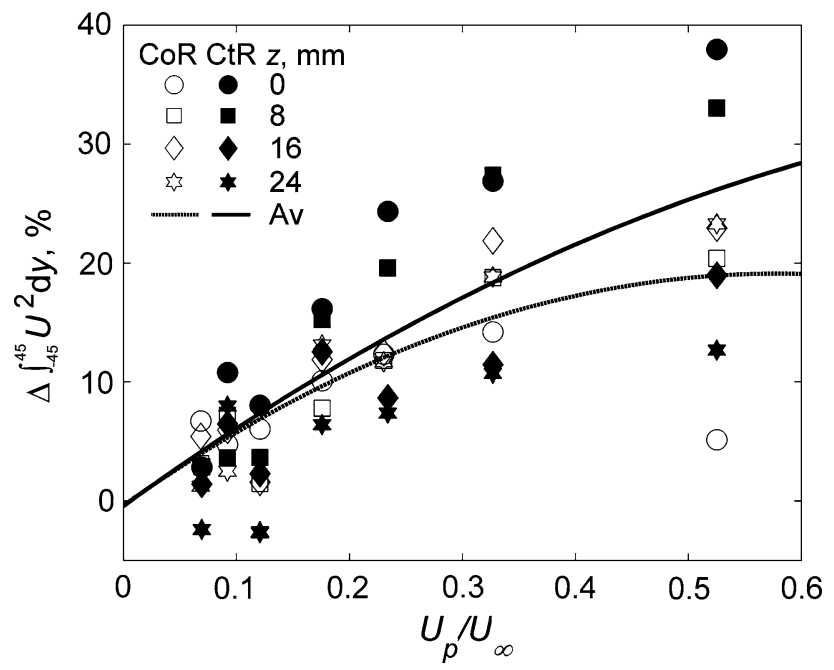

Fig. 25 Increase in streamwise momentum at $x=180 \mathrm{~mm}$ by CoR and CtR DBD-VGs

\section{Conclusions}

In this experimental investigation, we have demonstrated that yawed DBD plasma actuators can be used to create streamwise vortices. DBD-VGs have several advantages over vane-type vortex generators or vortex generator jets: they can be rapidly switched on and off, they should be without drag penalty when not in operation and they do not require ducting or machining of holes. We also expect that DBD-VGs should not need as accurate placement as DBDs oriented to produce a force with the flow (i.e. a spanwiseoriented upper electrode), which is the normal configuration for flow separation control applications. Additionally, these DBD-VGs can operate over a large streamwise distance which should be especially useful for cases where the separation point moves dynamically or is not known a priori.

The study of streamwise vortex development and its characteristics showed that the vortex circulation increased with plasma velocity ratio and actuator length and was maximised when the actuator was oriented to give force perpendicular to the flow. We believe that a circulation is set up above the DBD plasma due to mass continuity. This initiates a vortex with origin slightly above the wall and to the side of the DBD. The DBD actuator then creates a wall jet in the spanwise direction, which fuels the vortex from below. As this develops downstream, the wall jet becomes twisted by the oncoming boundary layer, lifted from the wall and then spirals around the vortex core. Co-rotating and counter-rotating vortex arrays were easily constructed, but the spanwise spacing should be carefully chosen to prevent unfavourable interactions with adjacent vortices whilst providing sufficient spanwise coverage to achieve efficient flow control.

Furthermore, the flow separation control ability of these DBD-VG arrays was demonstrated over a deflected ramp model. The flow could be completely reattached with effectiveness depending on array configuration and velocity ratio. We found that counter-rotating arrays were the most effective (directly downstream of the DBD) but were less homogenous than co-rotating arrays because CoR vortices naturally sweep across the span by their selfinduced velocity. It was demonstrated that flow separation control was still possible with plasma-to-free-stream velocity ratio as low as $7 \%\left(U_{\infty}=14.9 \mathrm{~m} / \mathrm{s}\right)$.

The work has been carried out as a part of PlasmAero program with funding from the European Community's Seventh Framework Programme FP7/2007-2013 under grant agreement no. 234201. An overview of PlasmAero can be found in Caruana (2010).

\section{References}

Angele K, Grewe F (2007) Instantaneous behaviour of streamwise vortices for turbulent boundary layer separation control. J Fluids Eng 129:226 
Benard N, Jolibois J, Touchard G, Moreau E (2008) A directional plasma-jet device generated by double DBD actuators-an active vortex generator for aerodynamic flow control. In: 4th AIAA flow control conference, AIAA 2008-3763

Bolitho M, Jacob J (2008) Thrust vectoring flow control using plasma synthetic jet actuators. In: 46th AIAA aerospace sciences meeting and exhibit, AIAA 2008-1368

Bushnell D (1992) Longitudinal vortex control-techniques and applications. Aeronaut J 96:293

Caruana D (2010) Plasmas for aerodynamic control. Plasma Phys Control Fusion 52:124045

Choi K-S, DeBisschop J-R, Clayton B (1998) Turbulent boundary-layer control by means of spanwise-wall oscillation. AIAA J 36:1157

Choi K-S, Jukes T, Whalley R (2010) Turbulent boundary-layer control with plasma actuators. Philos Trans R Soc A 369:1443

Compton D, Johnston J (1992) Streamwise vortex production by pitched and skewed jets in a turbulent boundary layer. AIAA J 30:640

Corke T, Enloe C, Wilkinson S (2010) Dielectric barrier discharge plasma actuators for flow control. Annu Rev Fluid Mech 42:505

Godard G, Stanislas M (2006a) Control of a decelerating boundary layer. Part 1: optimisation of passive vortex generators. Aerosp Sci Technol 10:181

Godard G, Stanislas M (2006b) Control of a decelerating boundary layer. Part 3: optimisation of round jet vortex generators. Aerosp Sci Technol 10:455

Godard G, Foucaut J, Stanislas M (2006) Control of a decelerating boundary layer. Part 2: optimisation of slotted jet vortex generators. Aerosp Sci Technol 10:394

Grundmann S, Tropea C (2008) Active cancellation of artificially introduced Tollmien-Schlichting waves using plasma actuators. Exp Fluids 44:795

Hanson R, Lavoie P, Naguib A, Morrison J (2010) Transient growth instability cancelation by a plasma actuator array. Exp Fluids 49:1339

Im S, Do H, Cappelli M (2010) Dielectric barrier discharge control of a turbulent boundary layer in a supersonic flow. Appl Phys Lett 97:041503

Johnston J (1999) Pitched and skewed vortex generator jets for control of turbulent boundary layer separation: a review. In: 3rd ASME/ JSME joint fluid engineering conference, FEDSM99-6917

Johnston J, Nishi M (1990) Vortex generator jets-means for flow separation control. AIAA J 28:989

Jukes T, Choi K-S (2009a) Control of unsteady flow separation over a circular cylinder using dielectric-barrier-discharge surface plasma. Phys Fluids 21:094106

Jukes T, Choi K-S (2009b) Flow control around a circular cylinder using pulsed dielectric barrier discharge surface plasma. Phys Fluids 21:084103

Jukes T, Choi K-S (2009c) Long lasting modifications to vortex shedding using a short plasma excitation. Phys Rev Lett 102:254501

Jukes T, Choi K-S (2009d) Active control of a cylinder wake using surface plasma. In: Braza M, Hourigan K (eds) IUTAM symposium on unsteady flow and their control, IUTAM Bookseries 14:539

Jukes T, Choi K-S, Johnson G, Scott S (2006) Turbulent drag reduction by surface plasma through spanwise flow oscillation. In: 3rd AIAA flow control conference, AIAA 2006-3693

Jukes T, Choi K-S, Segawa T, Yoshida H (2008) Jet flow induced by a surface plasma actuator. Proc IMechE Part I J Syst Control Eng 222:347

Khan Z, Johnston J (2000) On vortex generating jets. Int J Heat Fluid Flow 21:506
Kozlov A, Thomas F (2009) Active noise control of bluff-body flows using dielectric barrier discharge plasma actuators, In: 15th AIAA/CEAS aeroacoustics conference, AIAA 2009-3245

Lin J (2002) Review of research on low-profile vortex generators to control boundary-layer separation. Prog Aerosp Sci 38:389-420

Lögdberg O, Angele K, Alfredsson PH (2010) On the robustness of separation control by streamwise vortices. Eur J Mech B/Fluids 29:9

Mehta R, Bradshaw P (1988) Longitudinal vortices imbedded in turbulent boundary layers. Part 2. Vortex pair with 'common flow' upwards. J Fluid Mech 188:529

Moreau E (2007) Airflow control by non-thermal plasma actuators. J Phys D Appl Phys 40:605

Okita Y, Jukes T, Choi K-S, Nakamura K (2008) Flow reattachment over an airfoil using surface plasma actuator. In: 4th AIAA flow control conference, AIAA 2008-4203

Pauley W, Eaton J (1988) Experimental study of the development of longitudinal vortex pairs embedded in a turbulent boundary layer. AIAA J 26:816

Pearcey H (1961) Shock-induced separation and its prevention by design and boundary layer control. In: Lachmann G (ed) Boundary layer and flow control, vol 2. Pergamon Press, Oxford

Porter C, Abbas A, Cohen K, McLaughlin T, Enloe CL (2009) Spatially distributed forcing and jet vectoring with a plasma actuator. AIAA J 47:1368

Roth J, Sherman D, Wilkinson S (1998) Boundary layer flow control with a one atmosphere uniform glow discharge surface plasma. In: 36th AIAA aerospace sciences meeting and exhibit, AIAA 98-0328

Roy S, Wang C-C (2009) Bulk flow modification with horseshoe and serpentine plasma actuators, J. Phys D Appl Phys 42:032004

Schatzman D, Thomas F (2010) Turbulent boundary-layer separation control with single dielectric barrier discharge plasma actuators. AIAA J 48:1620

Schubauer G, Spangenberg W (1960) Forced mixing in boundary layers. J Fluid Mech 8:10

Segawa T, Yoshida H, Takekawa S, Jukes T, Choi K-S (2009) Generation of functional jet using DBD plasma actuator with facing linear electrodes. Theor Appl Mech Jpn 57:289

Shabaka I, Mehta R, Bradshaw P (1985) Longitudinal vortices imbedded in turbulent boundary layers. Part 1. Single vortex. J Fluid Mech 155:37

Taylor H D (1947) The elimination of diffuser separation by vortex generators. United Aircraft Corporation report No. R-4012-3

Thompson B, Whitelaw J (1985) Characteristics of a trailing-edge flow with turbulent boundary-layer separation. J Fluid Mech 157:305

Whalley R (2011) Turbulent boundary-layer control with DBD plasma actuators. PhD Thesis, University of Nottingham

Whalley R, Choi K-S (2010) Starting, travelling, and colliding vortices: dielectric-barrier-discharge plasma in quiescent air. Phys Fluids 22:091105

White F (1999) Fluid mechanics. McGraw-Hill, Singapore

You D, Wang M, Mittal R, Moin P (2006) Large-eddy simulations of longitudinal vortices embedded in a turbulent boundary layer. AIAA J 44:3032

Zhang X (1999) Counter-rotating vortices embedded in a turbulent boundary layer with inclined jets. AIAA J 37:1277

Zhang X (2000) An inclined rectangular jet in a turbulent boundary layer-vortex flow. Exp Fluids 28:344

Zhang X (2003) The evolution of co-rotating vortices in a canonical boundary layer with inclined jets. Phys Fluids 15:3693 